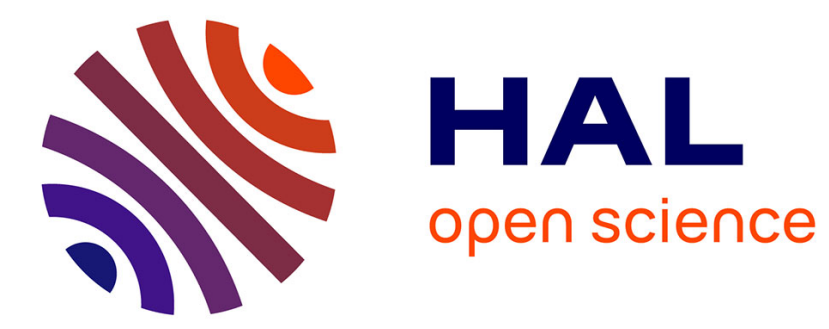

\title{
Gyrokinetic Vlasov equation in three dimensional setting. Second order approximation
}

\author{
Mihai Bostan
}

\section{To cite this version:}

Mihai Bostan. Gyrokinetic Vlasov equation in three dimensional setting. Second order approximation. 2009. hal-00431289

\author{
HAL Id: hal-00431289 \\ https://hal.science/hal-00431289 \\ Preprint submitted on 11 Nov 2009
}

HAL is a multi-disciplinary open access archive for the deposit and dissemination of scientific research documents, whether they are published or not. The documents may come from teaching and research institutions in France or abroad, or from public or private research centers.
L'archive ouverte pluridisciplinaire HAL, est destinée au dépôt et à la diffusion de documents scientifiques de niveau recherche, publiés ou non, émanant des établissements d'enseignement et de recherche français ou étrangers, des laboratoires publics ou privés. 


\title{
Gyrokinetic Vlasov equation in three dimensional setting. Second order approximation
}

\author{
Mihai Bostan *
}

(November 10, 2009)

\begin{abstract}
One of the main applications in plasma physics concerns the energy production through thermo-nuclear fusion. The controlled fusion requires the confinement of the plasma into a bounded domain and for this we appeal to the magnetic confinement. Several models exist for describing the evolution of strongly magnetized plasmas. The subject matter of this paper is to provide a rigorous derivation of the guiding-center approximation in the general three dimensional setting under the action of large stationary inhomogeneous magnetic fields. The first order corrections are computed as well : electric cross field drift, magnetic gradient drift, magnetic curvature drift, etc. The mathematical analysis relies on average techniques and ergodicity.
\end{abstract}

Keywords: Vlasov equation, Guiding-center approximation, Average operator.

AMS classification: $35 \mathrm{Q} 75,78 \mathrm{~A} 35$, 82D10.

\section{Introduction}

Motivated by the confinement fusion, many research programs in plasma physics focus on strongly magnetized plasmas. It concerns the evolution of a population of

*Laboratoire de Mathématiques de Besançon, UMR CNRS 6623, Université de Franche-Comté, 16 route de Gray, 25030 Besançon Cedex France. E-mail : mbostan@univ-fcomte.fr 
charged particles under the action of strong magnetic fields $\mathbf{B}^{\varepsilon}$ depending on some parameter $\varepsilon>0$. Using the kinetic description and neglecting the collisions we are led to the Vlasov equation

$$
\partial_{t} f^{\varepsilon}+\frac{p}{m} \cdot \nabla_{x} f^{\varepsilon}+q\left(E(t, x)+\frac{p}{m} \wedge \mathbf{B}^{\varepsilon}(x)\right) \cdot \nabla_{p} f^{\varepsilon}=0, \quad(t, x, p) \in \mathbb{R}_{+} \times \mathbb{R}^{3} \times \mathbb{R}^{3}
$$

with the initial condition

$$
f^{\varepsilon}(0, x, p)=f^{\text {in }}(x, p), \quad(x, p) \in \mathbb{R}^{3} \times \mathbb{R}^{3}
$$

where $f^{\varepsilon}=f^{\varepsilon}(t, x, p) \geq 0$ is the distribution function of the particles in the phase space $(x, p) \in \mathbb{R}^{3} \times \mathbb{R}^{3}, m$ is the particle mass and $q$ is the particle charge. Generally we close the Vlasov equation by adding equations for the electro-magnetic field $\left(E, \mathbf{B}^{\varepsilon}\right)$ (i.e., the Maxwell equations or the Poisson equation). Here we consider only the linear problem (1), (2) assuming that the magnetic field is stationary, divergence free and that the electric field derives from a given electric potential $E(t)=-\nabla_{x} \phi(t)$. We investigate the asymptotic behaviour of (1) when the magnetic field becomes large

$$
\mathbf{B}^{\varepsilon}(x)=\frac{\mathbf{B}(x)}{\varepsilon}, \quad \mathbf{B}(x)=B(x) b(x), \quad \operatorname{div}_{x}(B b)=0, \quad 0<\varepsilon<<1
$$

for some scalar positive function $B(x)$ and some field of unitary vectors $b(x)$. We assume that $B, b$ are smooth. Clearly the dynamics of the particles is dominated by the transport operator parallel to $q B(x)\left(\frac{p}{m} \wedge b(x)\right) \cdot \nabla_{p}$. Assuming that $\varepsilon$ is small enough we may expect that expansion like $f^{\varepsilon}=f+\varepsilon f^{1}+\varepsilon^{2} f^{2}+\ldots$ holds true and letting $\varepsilon \searrow 0$ it is easily seen that the leading order term belongs to the kernel of $\mathcal{T}=q B(x)\left(\frac{p}{m} \wedge b(x)\right) \cdot \nabla_{p}$. Notice that a family of independent invariants for $\mathcal{T}$ is given by $x,|p \wedge b(x)|, p \cdot b(x)$ and therefore the constraint $\mathcal{T} f=0$ is equivalent to

$$
f(t, x, p)=g(t, x, r=|p \wedge b(x)|, z=p \cdot b(x)) .
$$

Actually, plugging the above ansatz in (1) gives at the lowest order the divergence constraint $\mathcal{T} f=0$ and to the next order the evolution equation

$$
\partial_{t} f+\frac{p}{m} \cdot \nabla_{x} f+q E(t, x) \cdot \nabla_{p} f+\mathcal{T} f^{1}=0
$$

The key point is how to close (3) with respect to the first order fluctuation density $f^{1}$. The idea is to project on the kernel of $\mathcal{T}$ by observing that the range of $\mathcal{T}$ is orthogonal 
to its kernel. Indeed, this will give a well-posed mathematical model, since we already know that $f$ belongs to the kernel of $\mathcal{T}$. The computations considerably simplify if we observe that the orthogonal projection on $\operatorname{ker} \mathcal{T}$ is equivalent to averaging along the characteristic flow associated to $\mathcal{T}$. The rigorous construction of the average operator (sometimes called by physicists the gyro-average operator in the context of gyrokinetic models) essentially relies on ergodic theory i.e., von Neumann's ergodic theorem [19] pp. 57. Employing this method we derive rigorously the guiding-center approximation in the three dimensional setting and we obtain the following Vlasov equation for the leading order particle density

$$
\partial_{t} f+b(x) \otimes b(x) \frac{p}{m} \cdot \nabla_{x} f+\left(q b(x) \otimes b(x) E+\omega(x, p){ }^{\perp} p\right) \cdot \nabla_{p} f=0
$$

where $b(x)$ is the unitary vector field parallel to the magnetic field, the frequency $\omega(x, p)$ is given by

$$
\omega(x, p)=\frac{|p \wedge b(x)|}{2 m} \operatorname{div}_{x} b-\frac{(p \cdot b(x))}{m}\left(\frac{\partial b}{\partial x} b(x) \cdot \frac{p}{|p \wedge b(x)|}\right), p \wedge b(x) \neq 0
$$

and for any $(x, p)$ such that $p \wedge b(x) \neq 0$ the symbol ${ }^{\perp} p$ stands for the orthogonal momentum to $p$ in the plan determined by $b(x)$ and $p$ such that its coordinate along $b(x)$ is positive

$$
{ }^{{ }^{\perp}} p=|p \wedge b(x)| b(x)-(p \cdot b(x)) \frac{p-(p \cdot b(x)) b(x)}{|p \wedge b(x)|} .
$$

At the lowest order the particles are advected along the magnetic lines and only the parallel (with respect to $b$ ) electric field accelerates the particles. The plasma is confined along the magnetic lines and the transport operator in (1) becomes, in the limit $\varepsilon \searrow 0$

$$
A_{x} \cdot \nabla_{x}+A_{p} \cdot \nabla_{p}=b(x) \otimes b(x) \frac{p}{m} \cdot \nabla_{x}+\left(q b(x) \otimes b(x) E+\omega(x, p){ }^{\perp} p\right) \cdot \nabla_{p} .
$$

But orthogonal drifts are expected at the next order. More general we investigate higher order approximations for (1), leading to a transport operator which takes into account the first order corrections

$$
\left(A_{x}+\varepsilon A_{x}^{1}\right) \cdot \nabla_{x}+\left(A_{p}+\varepsilon A_{p}^{1}\right) \cdot \nabla_{p}
$$

Among these corrections we recover the electric cross field drift, the magnetic gradient drift and the magnetic curvature drift (cf. Theorem 5.2)

$$
A_{x}^{1}=v_{\wedge}+v_{\mathrm{GD}}+v_{\mathrm{CD}}+\ldots
$$


with

$$
v_{\wedge}=\frac{E \wedge b}{B}, \quad v_{\mathrm{GD}}=\frac{|p \wedge b|^{2}}{2 m^{2} \omega_{c}} \frac{b \wedge \nabla_{x} B}{B}, v_{\mathrm{CD}}=\frac{(p \cdot b)^{2}}{m^{2} \omega_{c}} b \wedge \partial_{x} b b, \omega_{c}=\frac{q B}{m} .
$$

The main point is that the particle dynamics evolves on two time scales $t$ and $s=t / \varepsilon$, the fast motion being associated to the large cyclotronic frequency $\frac{1}{\varepsilon} \frac{q B}{m}$. Accordingly the motion equations of the particles in (1) can be written

$$
\frac{\mathrm{d} X^{\varepsilon}}{\mathrm{d} t}=\frac{P^{\varepsilon}(t)}{m}, \frac{\mathrm{d} P^{\varepsilon}}{\mathrm{d} t}=q E\left(t, X^{\varepsilon}(t)\right)+\frac{1}{\varepsilon} \omega_{c}\left(X^{\varepsilon}(t)\right) P^{\varepsilon}(t) \wedge b\left(X^{\varepsilon}(t)\right)
$$

where

$$
X^{\varepsilon}(t)=X(t, t / \varepsilon)+\varepsilon X^{1}(t, t / \varepsilon)+\ldots, \quad P^{\varepsilon}(t)=P(t, t / \varepsilon)+\varepsilon P^{1}(t, t / \varepsilon)+\ldots
$$

Plugging the above ansatz in (5) one gets at the lowest order $\varepsilon^{-1}$

$$
\partial_{s} X=0, \quad \partial_{s} P=\omega_{c}(X) P \wedge b(X)
$$

and at the next order $\varepsilon^{0}$

$$
\begin{gathered}
\partial_{t} X+\partial_{s} X^{1}=\frac{P}{m} \\
\partial_{t} P+\partial_{s} P^{1}=q E(t, X)+\left(\nabla_{x} \omega_{c}(X) \cdot X^{1}\right) P \wedge b(X)+\omega_{c}(X)\left(P \wedge \partial_{x} b(X) X^{1}+P^{1} \wedge b(X)\right) .
\end{gathered}
$$

From equation (7) we deduce that $X,|P \wedge b(X)|, P \cdot b(X)$ depend only on the slow time scale

$$
X=X(t), \quad|P(t, s) \wedge b(X(t))|=R(t), \quad P(t, s) \cdot b(X(t))=Z(t)
$$

Moreover for any fixed $t$ we have, by the second equation in (7)

$$
\begin{aligned}
P(t, s) & =\cos \left(\omega_{c}(X(t)) s\right) b(X(t)) \wedge(P(t, 0) \wedge b(X(t))) \\
& +\sin \left(\omega_{c}(X(t)) s\right) P(t, 0) \wedge b(X(t)) \\
& +Z(t) b(X(t))
\end{aligned}
$$

and therefore, at any fixed time $t$ the momentum $P$ is $T_{c}(X(t))=2 \pi /\left|\omega_{c}(X(t))\right|$ periodic with respect to the fast variable $s$. Averaging the equation (8) with respect to $s$ over one cyclotronic period $T_{c}(X(t))$ one gets

$$
\frac{\mathrm{d} X}{\mathrm{~d} t}=\frac{Z(t)}{m} b(X(t)) .
$$


At the leading order the particles are advected along the magnetic lines. Notice that

$$
\partial_{s}(P \wedge b(X))=\partial_{s} P \wedge b(X)=\omega_{c}(X)(P \wedge b(X)) \wedge b(X)=\omega_{c}(X)((P \cdot b) b-P)
$$

implying that

$$
\partial_{s} X^{1}=\frac{P}{m}-\partial_{t} X=-\frac{\partial_{s}(P \wedge b(X))}{\omega_{c}(X) m} .
$$

Therefore $X^{1}+\frac{P \wedge b(X)}{m \omega_{c}(X)}$ is another invariant with respect to the fast motion. We can write

$$
X(t)+\varepsilon X^{1}(t, s)=X(t)+\varepsilon\left(X^{1}+\frac{P \wedge b(X)}{m \omega_{c}(X)}\right)-\varepsilon \frac{P \wedge b(X)}{m \omega_{c}(X)}
$$

saying that during a cyclotronic period $X(t)+\varepsilon X^{1}(t, t / \varepsilon) \approx X^{\varepsilon}(t)$ describes a circle of radius $\varepsilon \frac{R(t)}{m\left|\omega_{c}(X(t))\right|}$ into the plan orthogonal to $b(X(t))$. We compute now the acceleration along the magnetic lines by multiplying the equation $(9)$ by $b(X(t))$ and averaging over one cyclotronic period (here $\langle\cdot\rangle$ stands for the average with respect to $s$ over one period). For doing this observe that $\left\langle\partial_{s} P^{1} \cdot b(X(t))\right\rangle=0$ and

$$
\begin{aligned}
\left\langle\partial_{t} P \cdot b(X)\right\rangle & =\left\langle\partial_{t}(P \cdot b(X))-P \cdot \partial_{t} b(X)\right\rangle \\
& =\frac{\mathrm{d} Z}{\mathrm{~d} t}-\frac{Z(t)}{m}\left\langle P \cdot \partial_{x} b b(X)\right\rangle \\
& =\frac{\mathrm{d} Z}{\mathrm{~d} t}-\frac{Z^{2}(t)}{m}\left(b(X) \cdot \partial_{x} b b(X)\right) \\
& =\frac{\mathrm{d} Z}{\mathrm{~d} t} .
\end{aligned}
$$

The average contribution of the electric force during a cyclotronic period is clearly $q E(t, X(t)) \cdot b(X(t))$. It remains to compute the average contribution of the Laplace force. Notice that only the variation of the magnetic field direction accelerates the particles along the magnetic lines. Since $X^{1}+(P \wedge b(X)) /\left(m \omega_{c}(X)\right)$ is invariant with respect to the fast motion and $\operatorname{div}_{x}(B b)=b \cdot \nabla_{x} B+B \operatorname{div}_{x} b=0$ we can write

$$
\begin{aligned}
\left\langle\omega_{c}(X)\left(P \wedge \partial_{x} b(X) X^{1}\right) \cdot b(X)\right\rangle & =\frac{1}{m}\left\langle\partial_{x} b(X):(P \wedge b(X)) \otimes(P \wedge b(X))\right\rangle \\
& =\frac{|P \wedge b(X)|^{2}}{2 m}\left(\partial_{x} b:(I-b(X) \otimes b(X))\right) \\
& =\frac{R^{2}(t)}{2 m} \operatorname{div}_{x} b \\
& =-\frac{R^{2}(t)}{2 m B(X)} \nabla_{x} B \cdot b(X) .
\end{aligned}
$$


We have obtained the diamagnetic force $-\mu\left(\nabla_{x} B \cdot b\right)$ where $\mu(x, p)=\frac{|p \wedge b(x)|^{2}}{2 m B(x)}$ is the magnetic moment. Combining the above computations yields

$$
\frac{\mathrm{d} Z}{\mathrm{~d} t}=q E(t, X(t)) \cdot b(X(t))+\frac{R^{2}(t)}{2 m} \operatorname{div}_{x} b .
$$

Multiplying now the equation (9) by $P / m$ and averaging over one cyclotronic period we deduce

$$
\frac{\mathrm{d}}{\mathrm{d} t} \frac{R^{2}+Z^{2}}{2 m}+\left\langle\partial_{s} P^{1} \cdot \frac{P}{m}\right\rangle=q(E(t, X(t)) \cdot b(X(t))) \frac{Z(t)}{m}+\omega_{c}(X)\left\langle\left(P^{1} \wedge b(X)\right) \cdot \frac{P}{m}\right\rangle .
$$

Integrating by parts with respect to $s$ one gets

$$
\left\langle\partial_{s} P^{1} \cdot P\right\rangle=\omega_{c}(X)\left\langle\left(P^{1} \wedge b(X)\right) \cdot \frac{P}{m}\right\rangle .
$$

Therefore the time variation of the cyclotronic momentum $R(t)$ is given by

$$
\frac{R(t)}{m} \frac{\mathrm{d} R}{\mathrm{~d} t}+\frac{Z(t)}{m} \frac{\mathrm{d} Z}{\mathrm{~d} t}=q(E(t, X(t)) \cdot b(X(t))) \frac{Z(t)}{m} .
$$

Combining with (13) we obtain

$$
\frac{\mathrm{d} R}{\mathrm{~d} t}=-\frac{Z(t) R(t)}{2 m} \operatorname{div}_{x} b
$$

The dynamics of the particles with respect to the slow time scale in the phase space $(x, r, z)$ is given by $(11),(14),(13)$ leading to the limit model

$$
\partial_{t} g+\frac{z}{m} b(x) \cdot \nabla_{x} g-\frac{z r}{2 m} \operatorname{div}_{x} b \partial_{r} g+\left(q E(t, x) \cdot b(x)+\frac{r^{2}}{2 m} \operatorname{div}_{x} b\right) \partial_{z} g=0
$$

which is equivalent to (4) through the density change $f(t, x, p)=g(t, x,|p \wedge b(x)|, p$. $b(x))$. The derivation of the second order approximation for (1) follows by employing similar techniques. Nevertheless it is a much difficult task, which requires complex computations, eventually the choice of appropriate coordinate system.

The nonlinear gyrokinetic theory of the Vlasov-Maxwell equations can be carried out by appealing to Lagrangian and Hamiltonian methods [9], [16], [17]. It is also possible to follow the general method of multiple time scale or averaging perturbation developped in [1]. For a unified treatment of the main physical ideas and theoretical methods that have emerged on magnetic plasma confinement we refer to [15].

The guiding-center approximation for the Vlasov-Maxwell system was studied in [3] by the modulated energy method, see also [5], [7] for other results obtained by this 
method. The analysis of the Vlasov or Vlasov-Poisson equations with large external magnetic field have been carried out in [10], [12], [6], [11], [13]. The numerical approximation of the gyrokinetic models has been performed in [14] using semi-Lagrangian schemes. Other methods are based on the water bag representation of the distribution function: the full kinetic Vlasov equation is reduced to a set of hydrodynamic equations. This technique has been successefully applied to gyrokinetic models [18].

Our paper is organized as follows. In Section 2 we introduce the average operator and list its mathematical properties : orthogonal decomposition of $L^{2}$ functions into zero average functions and invariant functions along the characteristic flow, Poincaré inequality, etc. Section 3 is devoted to the derivation of the guiding-center approximation. This model is still a Vlasov equation. We investigare its conservative form and the geometry of its trajectories. We clearly identify invariants (magnetic moment, total energy) which allow us to reduce the dimension of the phase space. The asymptotic behaviour is studied in Section 4. We obtain both weak and strong convergence results. Section 5 is devoted to the second order approximation. One of the key points is to analyze the commutation properties between the average operator and first order differential operators.

\section{Average operator}

The main tool of our study is the average operator, which corresponds to the advection field dominating the transport operator in (1). For simplicity we work in the $L^{2}\left(\mathbb{R}^{3} \times \mathbb{R}^{3}\right)$ framework but similar analysis can be carried out in any Lebesgue space

$$
\begin{gathered}
\mathcal{T} u=\operatorname{div}_{p}\left(\omega_{c}(x) u p \wedge b(x)\right), \omega_{c}(x)=\frac{q B(x)}{m} \\
\mathrm{D}(\mathcal{T})=\left\{u(x, p) \in L^{2}\left(\mathbb{R}^{3} \times \mathbb{R}^{3}\right): \operatorname{div}_{p}\left(\omega_{c}(x) u p \wedge b(x)\right) \in L^{2}\left(\mathbb{R}^{3} \times \mathbb{R}^{3}\right)\right\} .
\end{gathered}
$$

We denote by $\|\cdot\|$ the standard norm of $L^{2}\left(\mathbb{R}^{3} \times \mathbb{R}^{3}\right)$. Notice that the above operator is local in $x$ i.e., if $u \in \mathrm{D}(\mathcal{T})$ then for a.a. $x \in \mathbb{R}^{3}$ we have

$$
u(x, \cdot) \in L^{2}\left(\mathbb{R}^{3}\right): \operatorname{div}_{p}\left(\omega_{c}(x) u(x, \cdot) p \wedge b(x)\right) \in L^{2}\left(\mathbb{R}^{3}\right) .
$$

We denote by $(X, P)(s ; x, p)$ the characteristics associated to $\omega_{c}(x)(p \wedge b(x)) \cdot \nabla_{p}$

$$
\frac{\mathrm{d} X}{\mathrm{~d} s}=0, \quad \frac{\mathrm{d} P}{\mathrm{~d} s}=\omega_{c}(X(s)) P(s) \wedge b(X(s)), \quad(X, P)(0)=(x, p) .
$$


Obviously $X(s)=x$ for any $s$ and by taking the scalar product of the second equation in (15) with $P(s)$ and $b(x)$ we deduce that $|P(s)|^{2}=|p|^{2}$ and $b(x) \cdot P(s)=b(x) \cdot p$ implying also that $|b(x) \wedge P(s)|=|b(x) \wedge p|$. If the initial conditions satisfy $b(x) \wedge p=0$ then clearly $P(s ; x, p)=(b(x) \cdot p) b(x)$. If $b(x) \wedge p \neq 0$ we consider the positive oriented basis of $\mathbb{R}^{3}$

$$
\tilde{\mathcal{B}}(x)=\left\{\tilde{e}_{1}(x)=\frac{b(x) \wedge(p \wedge b(x))}{|b(x) \wedge p|}, \quad \tilde{e}_{2}(x)=\frac{b(x) \wedge p}{|b(x) \wedge p|}, \quad \tilde{e}_{3}(x)=b(x)\right\} .
$$

Denoting $\left(\tilde{P}_{1}, \tilde{P}_{2}, \tilde{P}_{3}\right)(s)$ the coordinates of $P(s)$ in the basis $\tilde{\mathcal{B}}(x)$ we obtain the equations

$$
\frac{\mathrm{d} \tilde{P}_{1}}{\mathrm{~d} s}=\omega_{c}(x) \tilde{P}_{2}(s), \quad \frac{\mathrm{d} \tilde{P}_{2}}{\mathrm{~d} s}=-\omega_{c}(x) \tilde{P}_{1}(s), \quad \frac{\mathrm{d} \tilde{P}_{3}}{\mathrm{~d} s}=0
$$

and therefore $\tilde{P}_{3}(s)=\tilde{P}_{3}(0)$

$\tilde{P}_{1}(s)=\cos \left(\omega_{c}(x) s\right) \tilde{P}_{1}(0)+\sin \left(\omega_{c}(x) s\right) \tilde{P}_{2}(0), \quad \tilde{P}_{2}(s)=-\sin \left(\omega_{c}(x) s\right) \tilde{P}_{1}(0)+\cos \left(\omega_{c}(x) s\right) \tilde{P}_{2}(0)$.

Taking into account the formula $p=b(x) \wedge(p \wedge b(x))+(b(x) \cdot p) b(x)$ we deduce that $\tilde{P}_{1}(0)=|p \wedge b(x)|, \tilde{P}_{2}(0)=0, \tilde{P}_{3}(0)=(p \cdot b(x))$ and finally

$$
P(s ; x, p)=\cos \left(\omega_{c}(x) s\right) b(x) \wedge(p \wedge b(x))+\sin \left(\omega_{c}(x) s\right) p \wedge b(x)+(b(x) \cdot p) b(x) .
$$

Notice that the above formula holds also true in the case $p \wedge b(x)=0$. The motions $(X, P)(s ; x, p)$ are $T_{c}(x)=\frac{2 \pi}{\left|\omega_{c}(x)\right|}$ periodic for any initial condition $(x, p) \in \mathbb{R}^{3} \times \mathbb{R}^{3}$. We introduce the average operator cf. [4]

$$
\begin{aligned}
\langle u\rangle(x, p) & =\frac{1}{T_{c}(x)} \int_{0}^{T_{c}(x)} u(X(s ; x, p), P(s ; x, p)) \mathrm{d} s \\
& =\frac{1}{2 \pi} \int_{S(x)} u(x,|p \wedge b(x)| \omega+(p \cdot b(x)) b(x)) \mathrm{d} \omega
\end{aligned}
$$

for any function $u \in L^{2}\left(\mathbb{R}^{3} \times \mathbb{R}^{3}\right)$, where $S(x)=\left\{\omega \in S^{2}: b(x) \cdot \omega=0\right\}$.

Proposition 2.1 The average operator is linear continuous. Moreover it coincides with the orthogonal projection on the kernel of $\mathcal{T}$ i.e.,

$$
\langle u\rangle \in \operatorname{ker} \mathcal{T}: \int_{\mathbb{R}^{3}} \int_{\mathbb{R}^{3}}(u-\langle u\rangle) \varphi \mathrm{d} p \mathrm{~d} x=0, \quad \forall \varphi \in \operatorname{ker} \mathcal{T} .
$$

Proof. For any function $u \in L^{2}\left(\mathbb{R}^{3} \times \mathbb{R}^{3}\right)$ we have for a.a. $x \in \mathbb{R}^{3}$

$$
|\langle u\rangle|^{2}(x, p) \leq \frac{1}{T_{c}(x)} \int_{0}^{T_{c}(x)} u^{2}(x, P(s ; x, p)) \mathrm{d} s .
$$


Taking into account that for any $x \in \mathbb{R}^{3}$ the map $p \rightarrow P(s ; x, p)$ is measure preserving one gets

$$
\int_{\mathbb{R}^{3}} \int_{\mathbb{R}^{3}}\langle u\rangle^{2}(x, p) \mathrm{d} p \mathrm{~d} x \leq \int_{\mathbb{R}^{3}} \int_{\mathbb{R}^{3}} u^{2}(x, p) \mathrm{d} p \mathrm{~d} x
$$

saying that $\langle\cdot\rangle \in \mathcal{L}\left(L^{2}\left(\mathbb{R}^{3} \times \mathbb{R}^{3}\right), L^{2}\left(\mathbb{R}^{3} \times \mathbb{R}^{3}\right)\right)$ and $\|\langle\cdot\rangle\|_{\mathcal{L}\left(L^{2}\left(\mathbb{R}^{3} \times \mathbb{R}^{3}\right), L^{2}\left(\mathbb{R}^{3} \times \mathbb{R}^{3}\right)\right)} \leq 1$. It is well known that the kernel of $\mathcal{T}$ is given by the functions in $L^{2}$ invariant along the characteristics (15). Therefore we have

$$
\operatorname{ker} \mathcal{T}=\left\{u \in L^{2}\left(\mathbb{R}^{3} \times \mathbb{R}^{3}\right): \exists v \text { such that } u(x, p)=v(x,|p \wedge b(x)|,(p \cdot b(x)))\right\}
$$

Notice that for any $u \in L^{2}\left(\mathbb{R}^{3} \times \mathbb{R}^{3}\right)$ its average $\langle u\rangle$ depends only on $x,|p \wedge b(x)|,(p \cdot b(x))$. Therefore $\langle u\rangle \in \operatorname{ker} \mathcal{T}$. Pick a function $\varphi \in \operatorname{ker} \mathcal{T}$ i.e.,

$$
\exists \psi: \varphi(x, p)=\psi(x,|p \wedge b(x)|,(p \cdot b(x))) \in L^{2}\left(\mathbb{R}^{3} \times \mathbb{R}^{3}\right)
$$

and let us compute $I=\int_{\mathbb{R}^{3}} \int_{\mathbb{R}^{3}}(u-\langle u\rangle) \varphi \mathrm{d} p \mathrm{~d} x$. Using cylindrical coordinates along $b(x)$ axis yields

$$
I=\int_{\mathbb{R}^{3}} \int_{\mathbb{R}} \int_{\mathbb{R}_{+}} \psi(x, r, z)\left(\int_{S(x)} u(x, r \omega+z b(x)) \mathrm{d} \omega-2 \pi\langle u\rangle\right) r \mathrm{~d} r \mathrm{~d} z \mathrm{~d} x=0
$$

and therefore $\langle u\rangle=\operatorname{Proj}_{\text {ker } \mathcal{T}} u$ for any $u \in L^{2}\left(\mathbb{R}^{3} \times \mathbb{R}^{3}\right)$. In particular $\langle u\rangle=u$ for any $u \in \operatorname{ker} \mathcal{T}$ and $\|\langle\cdot\rangle\|_{\mathcal{L}\left(L^{2}\left(\mathbb{R}^{3} \times \mathbb{R}^{3}\right), L^{2}\left(\mathbb{R}^{3} \times \mathbb{R}^{3}\right)\right)}=1$.

For further use we inquire now about the solvability of $\mathcal{T} u=v$. It is easily seen that if $\mathcal{T} u=v$ is solvable (i.e., $v \in$ Range $\mathcal{T}$ ) then $\langle v\rangle=0$. Indeed, using the variational characterization of the average operator, we have for any function $\varphi \in \operatorname{ker} \mathcal{T}$

$$
\int_{\mathbb{R}^{3}} \int_{\mathbb{R}^{3}}(v-0) \varphi \mathrm{d} p \mathrm{~d} x=\int_{\mathbb{R}^{3}} \int_{\mathbb{R}^{3}} \mathcal{T} u \varphi \mathrm{d} p \mathrm{~d} x=-\int_{\mathbb{R}^{3}} \int_{\mathbb{R}^{3}} u \mathcal{T} \varphi \mathrm{d} p \mathrm{~d} x=0
$$

saying that $\langle v\rangle=0$. Generally we can prove that $\operatorname{ker}\langle\cdot\rangle=\overline{\text { Range } \mathcal{T}}$. Indeed, since $\langle\cdot\rangle=\operatorname{Proj}_{\text {ker } \mathcal{T}}$ and $\mathcal{T}^{\star}=-\mathcal{T}$ we have

$$
\operatorname{ker}\langle\cdot\rangle=(\operatorname{ker} \mathcal{T})^{\perp}=\left(\operatorname{ker} \mathcal{T}^{\star}\right)^{\perp}=\overline{\text { Range } \mathcal{T}}
$$

Moreover we have the orthogonal decomposition of $L^{2}\left(\mathbb{R}^{3} \times \mathbb{R}^{3}\right)$ into invariant functions along the characteristics (15) and zero average functions

$$
L^{2}\left(\mathbb{R}^{3} \times \mathbb{R}^{3}\right)=\operatorname{ker} \mathcal{T}^{\star} \oplus\left(\operatorname{ker} \mathcal{T}^{\star}\right)^{\perp}=\operatorname{ker} \mathcal{T} \oplus \overline{\text { Range } \mathcal{T}}=\operatorname{ker} \mathcal{T} \oplus \operatorname{ker}\langle\cdot\rangle .
$$

It happens that under additional hypotheses the range of $\mathcal{T}$ is closed, leading to the equality Range $\mathcal{T}=\operatorname{ker}\langle\cdot\rangle$. The key point here is the Poincaré inequality 
Proposition 2.2 We assume that $\inf _{x \in \mathbb{R}^{3}} B(x)>0$. Then $\mathcal{T}$ restricted to $\operatorname{ker}\langle\cdot\rangle$ is one to one map onto $\operatorname{ker}\langle\cdot\rangle$. Its inverse belongs to $\mathcal{L}(\operatorname{ker}\langle\cdot\rangle, \operatorname{ker}\langle\cdot\rangle)$ and we have the Poincaré inequality

$$
\|u\| \leq \frac{2 \pi}{\left|\omega_{0}\right|}\|\mathcal{T} u\|, \quad \omega_{0}=\frac{q}{m} \inf _{x \in \mathbb{R}^{3}} B(x) \neq 0
$$

for any $u \in \mathrm{D}(\mathcal{T}) \cap \operatorname{ker}\langle\cdot\rangle$.

Proof. By the previous computations we know that Range $\mathcal{T} \subset$ ker $\langle\cdot\rangle$. Assume now that $u \in \mathrm{D}(\mathcal{T}) \cap \operatorname{ker}\langle\cdot\rangle$ such that $\mathcal{T} u=0$. Since $\langle\cdot\rangle=\operatorname{Proj}_{\text {ker } \mathcal{T}}$ we have $u=\langle u\rangle=0$ saying that $\left.\mathcal{T}\right|_{\operatorname{ker}\langle\cdot\rangle}$ is injective. Consider now $v \in \operatorname{ker}\langle\cdot\rangle$ and let us prove that there is $u \in \operatorname{ker}\langle\cdot\rangle \cap \mathrm{D}(\mathcal{T})$ such that $\mathcal{T} u=v$. For any $\alpha>0$ there is a unique $u_{\alpha} \in \mathrm{D}(\mathcal{T})$ such that

$$
\alpha u_{\alpha}+\mathcal{T} u_{\alpha}=v
$$

Indeed it is easily seen that the solutions $\left(u_{\alpha}\right)_{\alpha>0}$ are given by

$$
u_{\alpha}(x, p)=\int_{\mathbb{R}_{-}} e^{\alpha s} v(x, P(s ; x, p)) \mathrm{d} s, \quad(x, p) \in \mathbb{R}^{3} \times \mathbb{R}^{3} .
$$

Applying the average operator to (18) yields $\left\langle u_{\alpha}\right\rangle=0$ for any $\alpha>0$. We are looking now for a bound of $\left(\left\|u_{\alpha}\right\|\right)_{\alpha>0}$. We introduce the function $V(s ; x, p)=\int_{s}^{0} v(x, P(\tau ; x, p)) \mathrm{d} \tau$. Notice that for any fixed $(x, p)$ the function $s \rightarrow V(s ; x, p)$ is $T_{c}(x)$ periodic, because $\langle v\rangle=0$ and thus $\|V(s ; x, \cdot)\|_{L^{2}\left(\mathbb{R}^{3}\right)} \leq T_{c}(x)\|v(x, \cdot)\|_{L^{2}\left(\mathbb{R}^{3}\right)}$ for any $s \in \mathbb{R}$. Integrating by parts we obtain

$$
u_{\alpha}(x, p)=-\int_{\mathbb{R}_{-}} e^{\alpha s} \partial_{s} V \mathrm{~d} s=\int_{\mathbb{R}_{-}} \alpha e^{\alpha s} V(s ; x, p) \mathrm{d} s
$$

implying that

$$
\left\|u_{\alpha}(x, \cdot)\right\|_{L^{2}\left(\mathbb{R}^{3}\right)} \leq \int_{\mathbb{R}_{-}} \alpha e^{\alpha s}\|V(s ; x, \cdot)\|_{L^{2}\left(\mathbb{R}^{3}\right)} \leq T_{c}(x)\|v(x, \cdot)\|_{L^{2}\left(\mathbb{R}^{3}\right)} \leq T_{0}\|v(x, \cdot)\|_{L^{2}\left(\mathbb{R}^{3}\right)},
$$

where $T_{0}=\frac{2 \pi}{\left|\omega_{0}\right|}$. After integration with respect to $x$ we obtain the uniform estimate $\left\|u_{\alpha}\right\| \leq T_{0}\|v\|$ for any $\alpha>0$. Extracting a sequence $\left(\alpha_{n}\right)_{n}$ such that $\lim _{n \rightarrow+\infty} \alpha_{n}=0$, $\lim _{n \rightarrow+\infty} u_{\alpha_{n}}=u$ weakly in $L^{2}\left(\mathbb{R}^{3} \times \mathbb{R}^{3}\right)$ we deduce easily that

$$
u \in \mathrm{D}(\mathcal{T}), \quad \mathcal{T} u=v, \quad\langle u\rangle=0, \quad\|u\| \leq T_{0}\|v\|
$$

saying that $\left(\left.\mathcal{T}\right|_{\operatorname{ker}\langle\cdot\rangle}\right)^{-1}$ is bounded linear operator and $\left\|\left(\left.\mathcal{T}\right|_{\operatorname{ker}\langle\cdot\rangle}\right)^{-1}\right\|_{\mathcal{L}(\operatorname{ker}\langle\cdot\rangle, \operatorname{ker}\langle\cdot\rangle)} \leq T_{0}$. 
Remark 2.1 For any function $v \in \operatorname{ker}\langle\cdot\rangle$ with compact support, the unique function $u \in \operatorname{ker}\langle\cdot\rangle \cap \mathrm{D}(\mathcal{T})$ such that $\mathcal{T} u=v$ has compact support. Indeed, assume that

$$
\operatorname{supp} v \subset\left\{(x, p) \in \mathbb{R}^{3} \times \mathbb{R}^{3}:|x| \leq L_{x},|p| \leq L_{p}\right\} \text {. }
$$

Since $|P(s ; x, p)|=|p|$ it is easily seen that

$$
\operatorname{supp} u_{\alpha} \subset\left\{(x, p) \in \mathbb{R}^{3} \times \mathbb{R}^{3}:|x| \leq L_{x},|p| \leq L_{p}\right\}, \quad \alpha>0
$$

and therefore the weak limit $u=\lim _{n \rightarrow+\infty} u_{\alpha_{n}}$ in $L^{2}\left(\mathbb{R}^{3} \times \mathbb{R}^{3}\right)$ satisfies $u \in \operatorname{ker}\langle\cdot\rangle \cap \mathrm{D}(\mathcal{T})$, $\mathcal{T} u=v$ and

$$
\operatorname{supp} u \subset\left\{(x, p) \in \mathbb{R}^{3} \times \mathbb{R}^{3}:|x| \leq L_{x},|p| \leq L_{p}\right\} \text {. }
$$

Corollary 2.1 Under the hypotheses of Proposition 2.2 assume that the function $v$ belongs to $W^{1, \infty}\left([0, T] ; L^{2}\left(\mathbb{R}^{3} \times \mathbb{R}^{3}\right)\right)$ such that $\langle v(t)\rangle=0$ for any $t \in[0, T]$. Then $\mathcal{T}^{-1} v$ belongs to $W^{1, \infty}\left([0, T] ; L^{2}\left(\mathbb{R}^{3} \times \mathbb{R}^{3}\right)\right)$ and we have

$$
\left\|\mathcal{T}^{-1} v\right\|_{W^{1, \infty}\left([0, T] ; L^{2}\left(\mathbb{R}^{3} \times \mathbb{R}^{3}\right)\right)} \leq T_{0}\|v\|_{W^{1, \infty}\left([0, T] ; L^{2}\left(\mathbb{R}^{3} \times \mathbb{R}^{3}\right)\right)}
$$

Proof. For any $t \in[0, T]$ we denote by $u(t)$ the unique function of $\operatorname{ker}\langle\cdot\rangle \cap D(\mathcal{T})$ such that $\mathcal{T} u(t)=v(t)$. By Proposition 2.2 we deduce that

$$
\|u\|_{L^{\infty}\left([0, T] ; L^{2}\left(\mathbb{R}^{3} \times \mathbb{R}^{3}\right)\right)} \leq T_{0}\|v\|_{L^{\infty}\left([0, T] ; L^{2}\left(\mathbb{R}^{3} \times \mathbb{R}^{3}\right)\right)}
$$

For any $t \in] 0, T[$ and $h>0$ small enough we have

$$
\|u(t+h)-u(t)\| \leq T_{0}\|v(t+h)-v(t)\| \leq T_{0} h\left\|v^{\prime}\right\|_{L^{\infty}\left([0, T] ; L^{2}\left(\mathbb{R}^{3} \times \mathbb{R}^{3}\right)\right)}
$$

implying that $u \in W^{1, \infty}\left([0, T] ; L^{2}\left(\mathbb{R}^{3} \times \mathbb{R}^{3}\right)\right)$ and

$$
\left\|u^{\prime}\right\|_{L^{\infty}\left([0, T] ; L^{2}\left(\mathbb{R}^{3} \times \mathbb{R}^{3}\right)\right)} \leq T_{0}\left\|v^{\prime}\right\|_{L^{\infty}\left([0, T] ; L^{2}\left(\mathbb{R}^{3} \times \mathbb{R}^{3}\right)\right)}
$$

One of the crucial points when studying the asymptotic behaviour of (1) is how to propagate the regularity through the map $\mathcal{T}^{-1}$. At the first sight this seems easily achieved by taking space/momentum derivatives in the equality $\mathcal{T} u=v$ and combining with the Poincaré inequality (17). Actually this arguments do not really work because the space/momentum derivatives may not commute with $\mathcal{T}$ and the average operator. 
Indeed, notice that the Poincaré inequality provides an estimate for some derivative of $u$, let say $\partial u$, only if $\langle\partial u\rangle=0$. Therefore, if $\partial$ and $\langle\cdot\rangle$ are not commuting, we may expect that $\langle\partial u\rangle \neq \partial\langle u\rangle=\partial 0=0$ and thus the Poincaré inequality can not be used. The above considerations lead naturally to derivatives along fields in involution with $\left(0, \omega_{c}(x) p \wedge b(x)\right) \in \mathbb{R}^{6}$ i.e., fields $c=c(x, p) \in \mathbb{R}^{6}$ such that the first order operator $c(x, p) \cdot \nabla_{x, p}$ commutes with $\mathcal{T}$. It was shown in [4] that the average operator is commuting with derivatives along any such field in involution and finally we show that $u$ inherits the regularity of $v$. It is also possible to appeal to a slightly different approach based on invariants [4]. We recall that a complete family of invariants for $\mathcal{T}$ is given by $\{x,|p \wedge b(x)|, p \cdot b(x)\}$. We will come back with more details about the propagation of regularity under the action of $\mathcal{T}^{-1}$ on zero average smooth functions, see Proposition 5.10.

\section{Limit model}

Using the properties of the average operator we investigate now the limit model of (1) when $\varepsilon \searrow 0$ by appealing to the method introduced in [4] for general transport problems. We perform our computations by assuming high enough smoothness. We will see that the limit model is still a Vlasov equation, whose well posedness follows by standard arguments. We emphasize that the method we employ here has been studied in detail in [4] (see also [2]) for linear transport problems with even more general dominant advection field, with characteristic flow not necessarily periodic. The starting point consists in using a Hilbert expansion

$$
f^{\varepsilon}=f+\varepsilon f^{1}+\varepsilon^{2} f^{2}+\ldots
$$

Plugging the above ansatz into (1) yields

$$
\begin{gathered}
\mathcal{T} f(t)=0 \\
\partial_{t} f+\frac{p}{m} \cdot \nabla_{x} f+q E(t, x) \cdot \nabla_{p} f+\mathcal{T} f^{1}(t)=0 \\
\partial_{t} f^{1}+\frac{p}{m} \cdot \nabla_{x} f^{1}+q E(t, x) \cdot \nabla_{p} f^{1}+\mathcal{T} f^{2}(t)=0
\end{gathered}
$$


From the constraint $\mathcal{T} f(t)=0$ we deduce that there is a function $g=g(t, x, r, z)$ such that

$$
f(t, x, p)=g(t, x,|p \wedge b(x)|,(p \cdot b(x))) .
$$

The time evolution of the dominant term $f$ is described by (21) but we need to close this equation with respect to the first order correction $f^{1}$. The key point here is to eliminate $\mathcal{T} f^{1}$ by using the equality $\operatorname{ker}\langle\cdot\rangle=$ Range $\mathcal{T}$. In this manner one gets the model

$$
\left\langle\partial_{t} f+\frac{p}{m} \cdot \nabla_{x} f+q E(t) \cdot \nabla_{p} f\right\rangle=0 .
$$

Certainly we need to transform (24) into a more readable form taking into account the symmetries in (23). Notice that, by construction, the time derivative and the average operator are commuting. Therefore, since $\langle f\rangle=f \in \operatorname{ker} \mathcal{T}$ we obtain

$$
\left\langle\partial_{t} f\right\rangle=\partial_{t}\langle f\rangle=\partial_{t} f
$$

We compute now the averages of the derivatives with respect to space and momentum. These computations become more complex due to the general geometry of the magnetic field. For the sake of the presentation we split them into separate lemmas.

Lemma 3.1 For any $a, b \in \mathbb{R}^{3}, \omega \in S^{2}$ we have

$$
(\omega \cdot a)(\omega \cdot b)+(\omega \wedge a) \cdot(\omega \wedge b)=a \cdot b
$$

In particular if $a \cdot b=0$ then $(\omega \wedge a) \cdot(\omega \wedge b)=-(\omega \cdot a)(\omega \cdot b)$.

Proof. For any $c \in \mathbb{R}^{3}$ we have $|c|^{2}=(\omega \cdot c)^{2}+|\omega \wedge c|^{2}$. The conclusion follows immediately by applying the previous formula with $c \in\{a, b, a+b\}$.

Lemma 3.2 Assume that $f(x, p)=g(x,|p \wedge b(x)|,(p \cdot b(x)))$. Then we have

$$
\left\langle\frac{p}{m} \cdot \nabla_{x} f\right\rangle=b(x) \otimes b(x) \frac{p}{m} \cdot \nabla_{x} g-\frac{(p \cdot b(x))|p \wedge b(x)|}{2 m} \operatorname{div}_{x} b \partial_{r} g+\frac{|p \wedge b(x)|^{2}}{2 m} \operatorname{div}_{x} b \partial_{z} g .
$$

Proof. For any $i \in\{1,2,3\}$ we have

$$
\partial_{x_{i}} f=\partial_{x_{i}} g+\partial_{r} g \frac{p \wedge b(x)}{|p \wedge b(x)|} \cdot\left(p \wedge \partial_{x_{i}} b\right)+\partial_{z} g\left(p \cdot \partial_{x_{i}} b\right) .
$$

Since $|b(x)|=1$ we have $\partial_{x_{i}} b \cdot b(x)=0$ and therefore by Lemma 3.1 one gets

$$
(b(x) \wedge p) \cdot\left(\partial_{x_{i}} b \wedge p\right)=-(b(x) \cdot p)\left(\partial_{x_{i}} b \cdot p\right)
$$


implying that

$$
\partial_{x_{i}} f=\partial_{x_{i}} g-\partial_{r} g \frac{(p \cdot b(x))}{|p \wedge b(x)|}\left(p \cdot \partial_{x_{i}} b\right)+\partial_{z} g\left(p \cdot \partial_{x_{i}} b\right) .
$$

We obtain the formula

$$
p \cdot \nabla_{x} f=p \cdot \nabla_{x} g-\partial_{r} g \frac{(p \cdot b(x))}{|p \wedge b(x)|}\left(\frac{\partial b}{\partial x}: p \otimes p\right)+\partial_{z} g\left(\frac{\partial b}{\partial x}: p \otimes p\right) .
$$

Notice that $\left(\nabla_{x} g\right)(x,|p \wedge b(x)|,(p \cdot b(x))) \in \operatorname{ker} \mathcal{T}$ and therefore

$$
\left\langle p \cdot \nabla_{x} g\right\rangle=\langle p\rangle \cdot \nabla_{x} g=(b(x) \cdot p)\left(b(x) \cdot \nabla_{x} g\right) .
$$

By direct computation we obtain that

$$
\langle p \otimes p\rangle(x, p)=\frac{1}{2}|p \wedge b(x)|^{2}(I-b(x) \otimes b(x))+(p \cdot b(x))^{2} b(x) \otimes b(x) .
$$

Taking into account that $\frac{\partial b}{\partial x}: b(x) \otimes b(x)=b(x) \cdot \nabla_{x} \frac{|b(x)|^{2}}{2}=0$ we deduce

$$
\left\langle\frac{\partial b}{\partial x}: p \otimes p\right\rangle=\frac{1}{2}|p \wedge b(x)|^{2} \operatorname{div}_{x} b
$$

and finally one gets

$$
\left\langle\frac{p}{m} \cdot \nabla_{x} f\right\rangle=b(x) \otimes b(x) \frac{p}{m} \cdot \nabla_{x} g-\frac{(p \cdot b(x))|p \wedge b(x)|}{2 m} \operatorname{div}_{x} b \partial_{r} g+\frac{|p \wedge b(x)|^{2}}{2 m} \operatorname{div}_{x} b \partial_{z} g .
$$

Lemma 3.3 Assume that $f=g(x,|p \wedge b(x)|,(p \cdot b(x)))$ and $E=E(x)$. Then we have

$$
\left\langle q E \cdot \nabla_{p} f\right\rangle=q(b(x) \cdot E(x)) \partial_{z} g .
$$

Proof. For any $i \in\{1,2,3\}$ we have

$$
\partial_{p_{i}} f=\partial_{r} g \frac{p \wedge b(x)}{|p \wedge b(x)|} \cdot\left(e_{i} \wedge b(x)\right)+\partial_{z} g b_{i}(x)
$$

where $\mathcal{B}=\left\{e_{1}, e_{2}, e_{3}\right\}$ is the canonical basis of $\mathbb{R}^{3}$. By Lemma 3.1 we know that

$$
(b(x) \cdot p)\left(b(x) \cdot e_{i}\right)+|b(x) \wedge p| \cdot\left(b(x) \wedge e_{i}\right)=p_{i}
$$

and therefore $|b(x) \wedge p| \cdot\left(b(x) \wedge e_{i}\right)=(p-(b(x) \cdot p) b(x))_{i}$. We obtain the formula

$$
\nabla_{p} f=\frac{\partial_{r} g}{|p \wedge b(x)|}(I-b(x) \otimes b(x)) p+\partial_{z} g b(x)
$$

and finally, since $\left(\partial_{r} g, \partial_{z} g\right)(x,|p \wedge b(x)|,(p \cdot b(x))), E(x) \in \operatorname{ker} \mathcal{T}$, one gets $\left\langle q E \cdot \nabla_{p} f\right\rangle=$ $q(b(x) \cdot E(x)) \partial_{z} g$. 
Combining the computations in Lemmas 3.2, 3.3 yields the following Vlasov equation in the phase space $(x, r, z) \in \mathbb{R}^{3} \times \mathbb{R}_{+} \times \mathbb{R}$

$$
\partial_{t} g+\frac{z}{m} b(x) \cdot \nabla_{x} g-\frac{z r}{2 m} \operatorname{div}_{x} b \partial_{r} g+\left(\frac{r^{2}}{2 m} \operatorname{div}_{x} b+q(b(x) \cdot E(t, x))\right) \partial_{z} g=0
$$

whose characteristics $(X, R, Z)(s ; t, x, r, z)$ are given by

$$
\begin{gathered}
\frac{\mathrm{d} X}{\mathrm{~d} s}=\frac{Z(s)}{m} b(X(s)) \\
\frac{\mathrm{d} R}{\mathrm{~d} s}=-\frac{Z(s) R(s)}{2 m} \operatorname{div}_{x} b(X(s)) \\
\frac{\mathrm{d} Z}{\mathrm{~d} s}=\frac{R(s)^{2}}{2 m} \operatorname{div}_{x} b(X(s))+q(b(X(s)) \cdot E(s, X(s))) \\
(X, R, Z)(t)=(x, r, z) .
\end{gathered}
$$

Certainly it is possible to write a Vlasov equation for the dominant distribution $f$. For this it is sufficient to express the derivatives of $g$ with respect to the derivatives of $f$. Recall that we have already obtained the formula $\partial_{t} f=\partial_{t} g$

$$
\nabla_{x} f=\nabla_{x} g-\partial_{r} g \frac{(p \cdot b(x))}{|p \wedge b(x)|}{ }^{t} \partial_{x} b p+\partial_{z} g^{t} \partial_{x} b p, \quad \nabla_{p} f=\partial_{r} g \frac{b(x) \wedge(p \wedge b(x))}{|p \wedge b(x)|}+\partial_{z} g b(x) .
$$

Accordingly the derivatives of $g$ write

$$
\begin{gathered}
\partial_{t} g=\partial_{t} f, \quad \partial_{z} g=b(x) \cdot \nabla_{p} f, \quad \partial_{r} g=\frac{b(x) \wedge(p \wedge b(x))}{|p \wedge b(x)|} \cdot \nabla_{p} f \\
\nabla_{x} g=\nabla_{x} f+\left(\frac{b(x) \wedge(p \wedge b(x))}{|p \wedge b(x)|} \cdot \nabla_{p} f\right) \frac{(p \cdot b(x))}{|p \wedge b(x)|}{ }^{t} \partial_{x} b p-\left(b(x) \cdot \nabla_{p} f\right)^{t} \partial_{x} b p
\end{gathered}
$$

leading to the following Vlasov equation

$$
\partial_{t} f+b(x) \otimes b(x) \frac{p}{m} \cdot \nabla_{x} f+\left(F_{\perp}+F_{\|}\right) \cdot \nabla_{p} f=0
$$

where

$$
F_{\perp}=-\omega(x, p)(p \cdot b(x)) \frac{b(x) \wedge(p \wedge b(x))}{|p \wedge b(x)|}, \quad F_{\|}=(\omega(x, p)|p \wedge b(x)|+q E(t, x) \cdot b(x)) b(x)
$$

and

$$
\omega(x, p)=\frac{|p \wedge b(x)|}{2 m} \operatorname{div}_{x} b-\frac{(p \cdot b(x))}{m}\left(\partial_{x} b b(x) \cdot \frac{p}{|p \wedge b(x)|}\right) .
$$

Observe that $F_{\perp}+F_{\|}=q b(x) \otimes b(x) E+\omega(x, p){ }^{\perp} p$ and thus (31) reduces to (4). Notice that the forces $F_{\perp}, F_{\|}$may become singular when the momentum $p$ is parallel to the 
magnetic field. Nevertheless the forces remain bounded around these singularities. Indeed, since ${ }^{t} \partial_{x} b b(x)=0$ we can write

$$
\left(\partial_{x} b b(x) \cdot \frac{p}{|p \wedge b(x)|}\right)=\left(\partial_{x} b b(x) \cdot \frac{b(x) \wedge(p \wedge b(x))}{|p \wedge b(x)|}\right)
$$

implying that the frequency $\omega(x, p)$ remains bounded (and therefore the forces $F_{\perp}, F_{\|}$ as well)

$$
|\omega(x, p)| \leq \frac{|p \wedge b(x)|}{2 m}\left|\operatorname{div}_{x} b\right|+\frac{|(p \cdot b(x))|}{m}\left|\partial_{x} b b(x)\right| .
$$

It remains to determine the initial condition for (31). For this we multiply (1) with $\eta(t) \varphi(x, p)$ where $\eta \in C_{c}^{1}\left(\mathbb{R}_{+}\right)$and $\varphi \in C_{c}^{1}\left(\mathbb{R}^{3} \times \mathbb{R}^{3}\right) \cap \operatorname{ker} \mathcal{T}$. We deduce the weak formulation

$$
\begin{aligned}
& -\int_{\mathbb{R}_{+}} \eta^{\prime}(t) \int_{\mathbb{R}^{3}} \int_{\mathbb{R}^{3}} f^{\varepsilon}(t, x, p) \varphi(x, p) \mathrm{d} p \mathrm{~d} x \mathrm{~d} t-\eta(0) \int_{\mathbb{R}^{3}} \int_{\mathbb{R}^{3}} f^{\varepsilon}(0, x, p) \varphi(x, p) \mathrm{d} p \mathrm{~d} x \\
& -\int_{\mathbb{R}_{+}} \eta(t) \int_{\mathbb{R}^{3}} \int_{\mathbb{R}^{3}} f^{\varepsilon}(t, x, p)\left(\frac{p}{m} \cdot \nabla_{x} \varphi+q E(t, x) \cdot \nabla_{p} \varphi\right) \mathrm{d} p \mathrm{~d} x \mathrm{~d} t=0 .
\end{aligned}
$$

Passing to the limit as $\varepsilon \searrow 0$ one gets

$$
\frac{\mathrm{d}}{\mathrm{d} t} \int_{\mathbb{R}^{3}} \int_{\mathbb{R}^{3}} f(t, x, p) \varphi(x, p) \mathrm{d} p \mathrm{~d} x=\int_{\mathbb{R}^{3}} \int_{\mathbb{R}^{3}} f(t, x, p)\left(\frac{p}{m} \cdot \nabla_{x} \varphi+q E(t, x) \cdot \nabla_{p} \varphi\right) \mathrm{d} p \mathrm{~d} x
$$

and

$$
\int_{\mathbb{R}^{3}} \int_{\mathbb{R}^{3}} f(0, x, p) \varphi(x, p) \mathrm{d} p \mathrm{~d} x=\lim _{t \searrow 0} \int_{\mathbb{R}^{3}} \int_{\mathbb{R}^{3}} f(t, x, p) \varphi(x, p) \mathrm{d} p \mathrm{~d} x=\int_{\mathbb{R}^{3}} \int_{\mathbb{R}^{3}} f^{\text {in }}(x, p) \varphi(x, p) \mathrm{d} p \mathrm{~d} x
$$

implying that

$$
\int_{\mathbb{R}^{3}} \int_{\mathbb{R}^{3}}\left(f^{\text {in }}(x, p)-f(0, x, p)\right) \varphi(x, p) \mathrm{d} p \mathrm{~d} x=0, \quad \forall \varphi \in \operatorname{ker} \mathcal{T}
$$

Since we already know that $f(0, \cdot, \cdot) \in \operatorname{ker} \mathcal{T}$ we deduce by Proposition 2.1 that

$$
f(0, x, p)=\left\langle f^{\text {in }}\right\rangle(x, p)=: g^{\text {in }}(x,|p \wedge b(x)|,(p \cdot b(x))), \quad(x, p) \in \mathbb{R}^{3} \times \mathbb{R}^{3}
$$

At this stage let us make some comments about the limit model (31), (33). The particles are advected only along the magnetic field lines and consequently there is no current in the orthogonal directions to the magnetic lines

$$
j(t, x)=q \int_{\mathbb{R}^{3}} f(t, x, p) \frac{p}{m} \mathrm{~d} p=q \int_{\mathbb{R}^{3}} f(t, x, p) \frac{\langle p\rangle}{m} \mathrm{~d} p=q \int_{\mathbb{R}^{3}} f(t, x, p) \frac{(p \cdot b(x))}{m} \mathrm{~d} p b(x) .
$$


Notice also that the electric field accelerates the particles only along the magnetic field lines. It can be shown that the constraint $\mathcal{T} f(t)=0$ is propagated by the Vlasov equation (31), i.e., $\mathcal{T} f(t)=0 \forall t>0$ provided that $\mathcal{T} f(0)=0$. For checking this let us introduce the characteristics $(X, P)(s ; t, x, p)$ associated to $(31)$

$$
\frac{\mathrm{d} X}{\mathrm{~d} s}=b(X(s)) \otimes b(X(s)) \frac{P(s)}{m}, \frac{\mathrm{d} P}{\mathrm{~d} s}=\left(F_{\perp}+F_{\|}\right)(s, X(s), P(s)), \quad(X, P)(t)=(x, p) .
$$

A straightforward computation shows that the quantities $X(s),|P(s) \wedge b(X(s))|,(P(s)$. $b(X(s)))$ satisfy the characteristic equations $(27),(28),(29)$ implying that

$$
(X,|P \wedge b(X)|,(P \cdot b(X)))(0 ; t, x, p)=(X, R, Z)(0 ; t, x,|p \wedge b(x)|,(p \cdot b(x))) .
$$

Therefore one gets

$$
\begin{aligned}
f(t, x, p) & =f(0, X(0 ; t, x, p), P(0 ; t, x, p)) \\
& =g^{\text {in }}(X(0 ; t, x, p),|P(0 ; t, x, p) \wedge b(X(0 ; t, x, p))|,(P(0 ; t, x, p) \cdot b(X(0 ; t, x, p)))) \\
& =g^{\text {in }}((X, R, Z)(0 ; t, x,|p \wedge b(x)|,(p \cdot b(x)))) \in \operatorname{ker} \mathcal{T} .
\end{aligned}
$$

By the above considerations we know that the problems (26), (31) are equivalent. Nevertheless, for the numerical point of view it is preferable to consider the problem (26) since its phase space $(x, r, z) \in\left(\mathbb{R}^{3} \times \mathbb{R}_{+} \times \mathbb{R}\right)$ has only 5 dimensions, whereas (31) is posed in a 6 dimensional phase space. At the first sight the resolution of (26) may require some conditions on the boundary $r=0$ at any time $t>0$. Actually this is not the case, as emphasized in the following proposition.

Proposition 3.1 We denote by $\mu=\mu(x, p)$ the magnetic moment

$$
\mu(x, p)=\frac{|p \wedge b(x)|^{2}}{2 m B(x)}=\frac{r^{2}}{2 m B(x)} .
$$

If the magnetic field is divergence free, then the magnetic moment is an invariant for (31), resp. (26). In particular the solution of (26) is given by

$$
g(t, x, r, z)= \begin{cases}g^{\text {in }}((X, R, Z)(0 ; t, x, r, z)), & \text { if }(t, x, r, z) \in \mathbb{R}_{+} \times \mathbb{R}^{3} \times \mathbb{R}_{+}^{\star} \times \mathbb{R} \\ g^{\text {in }}(\tilde{X}(0 ; t, x, z), 0, \tilde{Z}(0 ; t, x, z)), & \text { if }(t, x, r, z) \in \mathbb{R}_{+} \times \mathbb{R}^{3} \times\{0\} \times \mathbb{R}\end{cases}
$$

where $(\tilde{X}, \tilde{Z})$ solve

$$
\frac{\mathrm{d} \tilde{X}}{\mathrm{~d} s}=\frac{\tilde{Z}(s)}{m} b(\tilde{X}(s)), \quad \frac{\mathrm{d} \tilde{Z}}{\mathrm{~d} s}=q E(s, \tilde{X}(s)) \cdot b(\tilde{X}(s)), \quad(\tilde{X}, \tilde{Z})(t)=(x, z) .
$$


Proof. We perform the computations with respect to the coordinates $(x, r, z)$. We have

$$
\begin{aligned}
& \left(\partial_{t}+\frac{z}{m} b(x) \cdot \nabla_{x}-\frac{z r}{2 m} \operatorname{div}_{x} b \partial_{r}+\left(\frac{r^{2}}{2 m} \operatorname{div}_{x} b+q E(t, x) \cdot b(x)\right) \partial_{z}\right) \mu= \\
= & -\frac{r^{2} z}{2 m^{2} B^{2}(x)}\left(b(x) \cdot \nabla_{x} B+B(x) \operatorname{div}_{x} b\right) \\
= & -\frac{r^{2} z}{2 m^{2} B^{2}(x)} \operatorname{div}_{x}(B b)=0 .
\end{aligned}
$$

The invariance of the magnetic moment ensures that $R^{2}(s ; t, x, r, z)>0$ for any $s \geq 0$, $r>0$ and by the continuity of the application $s \rightarrow R(s ; t, x, r, z)$ we deduce that $R(s ; t, x, r, z)>0$ for any $s \geq 0, r>0$. In the case $r=0$ the same invariance guarantees that $R(s ; t, x, r, z)=0$ for any $s \geq 0$ and thus the characteristic equations for $(X, R, Z)$ reduce to that of $(\tilde{X}, \tilde{Z})$. Therefore the solution of $(26)$ is given by the formula (35).

Remark 3.1 If a charged particle situated at the point $x$ has momentum parallel to the magnetic field, i.e., $p \wedge b(x)=0$, then at any time $s$ the particle remains to the same magnetic line and its coordinates in the phase space satisfy $\frac{\mathrm{d} X}{\mathrm{~d} s}=\frac{b(X(s)) \cdot P(s)}{m} b(X(s)), \frac{\mathrm{d}}{\mathrm{d} s}(b(X(s)) \cdot P(s))=q E(s, X(s)) \cdot b(X(s)), P(s) \wedge b(X(s))=0$.

Remark 3.2 We recognize the expression of the diamagnetic force acting in the parallel direction to the magnetic field. The parallel component of the force $\omega(x, p){ }^{\perp} p$ is

$$
\frac{|p \wedge b(x)|^{2}}{2 m} \operatorname{div}_{x} b b(x)=\mu(x, p) B(x) \operatorname{div}_{x} b b(x)=-\mu(x, p)\left(\nabla_{x} B \cdot b(x)\right) b(x) .
$$

For further computations it is useful to write the equation (31) in conservative form. By direct calculus we obtain

$$
\operatorname{div}_{x}\left(b(x) \otimes b(x) \frac{p}{m}\right)+\operatorname{div}_{p}\left(F_{\perp}+F_{\|}\right)=\frac{(p \cdot b(x))^{2}}{|p \wedge b(x)|^{2}}\left(\partial_{x} b b(x) \cdot \frac{b(x) \wedge(p \wedge b(x))}{m}\right)
$$

and therefore (31) is equivalent to

$\partial_{t} f+\operatorname{div}_{x}\left(f b(x) \otimes b(x) \frac{p}{m}\right)+\operatorname{div}_{p}\left(f\left(F_{\perp}+F_{\|}\right)\right)=f \frac{(p \cdot b(x))^{2}}{|p \wedge b(x)|^{2}}\left(\partial_{x} b b(x) \cdot \frac{b(x) \wedge(p \wedge b(x))}{m}\right)$.

A direct consequence of the above conservative form (with zero average source term) is the balance of the total energy. 
Proposition 3.2 We have the kinetic energy balance

$$
\frac{\mathrm{d}}{\mathrm{d} t} \int_{\mathbb{R}^{3}} \int_{\mathbb{R}^{3}} f(t, x, p) \frac{|p|^{2}}{2 m} \mathrm{~d} p \mathrm{~d} x=\int_{\mathbb{R}^{3}} E(t, x) \cdot \int_{\mathbb{R}^{3}} q f(t, x, p) \frac{p}{m} \mathrm{~d} p \mathrm{~d} x, \quad t \in \mathbb{R}_{+} \cdot
$$

In particular si $E=-\nabla_{x} \phi$ satisfies the Poisson equation $-\Delta_{x} \phi=\frac{q}{\varepsilon_{0}} \int_{\mathbb{R}^{3}} f \mathrm{~d} p$ then the total energy (kinetic and electrostatic) is conserved

$$
\frac{\mathrm{d}}{\mathrm{d} t} \int_{\mathbb{R}^{3}} \int_{\mathbb{R}^{3}} f(t, x, p)\left(\frac{|p|^{2}}{2 m}+\frac{q \phi(t, x)}{2}\right) \mathrm{d} p \mathrm{~d} x=0 .
$$

Proof. Notice that the functions $f,(p \cdot b(x))^{2},|p \wedge b(x)|^{2},|p|^{2}$ belong to ker $\mathcal{T}$ and $\langle p \wedge b(x)\rangle=0$ implying that

$$
\int_{\mathbb{R}^{3}} \int_{\mathbb{R}^{3}} f(t, x, p) \frac{|p|^{2}}{2 m} \frac{(p \cdot b(x))^{2}}{|p \wedge b(x)|^{2}}\left(\partial_{x} b b(x) \cdot \frac{b(x) \wedge(p \wedge b(x))}{m}\right) \mathrm{d} p \mathrm{~d} x=0 .
$$

Therefore multiplying (37) by $\frac{|p|^{2}}{2 m}$ yields after integration over $\mathbb{R}^{3} \times \mathbb{R}^{3}$

$$
\begin{aligned}
\frac{\mathrm{d}}{\mathrm{d} t} \int_{\mathbb{R}^{3}} \int_{\mathbb{R}^{3}} f(t, x, p) \frac{|p|^{2}}{2 m} \mathrm{~d} p \mathrm{~d} x & =\int_{\mathbb{R}^{3}} \int_{\mathbb{R}^{3}} f(t, x, p) \frac{p}{m} \cdot\left(F_{\perp}+F_{\|}\right) \mathrm{d} p \mathrm{~d} x \\
& =\int_{\mathbb{R}^{3}} E(t, x) \cdot \int_{\mathbb{R}^{3}} q f(t, x, p) b(x) \otimes b(x) \frac{p}{m} \mathrm{~d} p \mathrm{~d} x \\
& =\int_{\mathbb{R}^{3}} E(t, x) \cdot \int_{\mathbb{R}^{3}} q f(t, x, p) \frac{p}{m} \mathrm{~d} p \mathrm{~d} x .
\end{aligned}
$$

The conservation of the total energy follows in standard manner by using the continuity and Poisson equations.

Remark 3.3 The Vlasov equation (31) can be written in conservative form. Indeed, by (36) we have

$$
\operatorname{div}_{x}\left(b(x) \otimes b(x) \frac{p}{m}\right)+\operatorname{div}_{p}\left(F_{\perp}+F_{\|}\right)=\mathcal{T} \lambda, \quad \lambda=-\frac{(p \cdot b(x))^{2}}{|p \wedge b(x)|^{2}}\left(\partial_{x} b b \cdot \frac{p \wedge b(x)}{m \omega_{c}}\right)
$$

and since $\mathcal{T} f=0$, the equation (31) is equivalent to

$$
\partial_{t} f+\operatorname{div}_{x}\left(f b(x) \otimes b(x) \frac{p}{m}\right)+\operatorname{div}_{p}\left(f\left(F_{\perp}+F_{\|}-\lambda \omega_{c}(x) p \wedge b(x)\right)\right)=0 .
$$

In Proposition 3.1 it was shown that the magnetic moment $\mu$ is an invariant for (26). As usual this allows us to reduce (26) to a transport problem depending on one parameter, posed in a 4 dimensional phase space. Indeed, the change of variable

$$
g(t, x, r, z)=k(t, x, \mu, z), \quad \mu=\frac{r^{2}}{2 m B(x)}
$$


leads to the problem

$\partial_{t} k+\frac{z}{m} b(x) \cdot \nabla_{x} k+\left(q E(t, x)-\mu \nabla_{x} B\right) \cdot b(x) \partial_{z} k=0, \quad(t, x, z) \in \mathbb{R}_{+} \times \mathbb{R}^{3} \times \mathbb{R}, \mu \in \mathbb{R}_{+}$.

Motivated by such reductions, it is worth searching for other invariants. By direct computation we check the invariance of the energy function.

Proposition 3.3 Assume that the electric potential is stationary i.e., $\partial_{t} \phi=0$. Then the energy $e=\frac{|p|^{2}}{2 m}+q \phi(x)=\frac{r^{2}+z^{2}}{2 m}+q \phi(x)$ is an invariant for (31), resp. (26).

\section{Asymptotic behaviour}

Our goal thereafter will be to give some details about the convergence as $\varepsilon \searrow 0$ of the solutions $\left(f^{\varepsilon}\right)_{\varepsilon>0}$ for (1), (2) towards the solution of (31), (33). First we focus on weak convergence results. Secondly we derive strong convergence results for well prepared initial data.

Theorem 4.1 Assume that $\left(f_{\varepsilon}^{\text {in }}\right)_{\varepsilon>0}$ converges weakly in $L^{2}\left(\mathbb{R}^{3} \times \mathbb{R}^{3}\right)$ as $\varepsilon \searrow 0$ to some function $f^{\text {in }} \in L^{2}\left(\mathbb{R}^{3} \times \mathbb{R}^{3}\right)$ and let us denote by $\left(f^{\varepsilon}\right)_{\varepsilon>0}$ the sequence of weak solutions of (1) with the initial conditions $\left(f_{\varepsilon}^{\text {in }}\right)_{\varepsilon>0}$. We suppose that $E \in L_{\mathrm{loc}}^{\infty}\left(\mathbb{R}_{+} ; L^{\infty}\left(\mathbb{R}^{3}\right)\right)^{3}, b \in$ $W^{1, \infty}\left(\mathbb{R}^{3}\right)^{3}$ and $\operatorname{div}_{x}(B b)=0$. Then $\left(f^{\varepsilon}\right)_{\varepsilon>0}$ converges weakly $\star$ in $L^{\infty}\left(\mathbb{R}_{+} ; L^{2}\left(\mathbb{R}^{3} \times \mathbb{R}^{3}\right)\right)$ as $\varepsilon \searrow 0$ to the weak solution of (31), (33).

Proof. It is a straightforward consequence of the computations in Lemmas 3.2, 3.3. We only sketch the main steps. Since the characteristic flow associated to the transport operator $\frac{p}{m} \cdot \nabla_{x}+q\left(E(t, x)+\frac{p}{m} \wedge \mathbf{B}^{\varepsilon}\right) \cdot \nabla_{p}$ is measure preserving we deduce that

$$
\int_{\mathbb{R}^{3}} \int_{\mathbb{R}^{3}}\left|f^{\varepsilon}(t, x, p)\right|^{2} \mathrm{~d} p \mathrm{~d} x=\int_{\mathbb{R}^{3}} \int_{\mathbb{R}^{3}}\left|f_{\varepsilon}^{\text {in }}(x, p)\right|^{2} \mathrm{~d} p \mathrm{~d} x, \quad t \in \mathbb{R}_{+}, \varepsilon>0
$$

and therefore, after extraction eventually, we can assume that $\left(f^{\varepsilon}\right)_{\varepsilon>0}$ converges weakly $\star$ in $L^{\infty}\left(\mathbb{R}_{+} ; L^{2}\left(\mathbb{R}^{3} \times \mathbb{R}^{3}\right)\right)$ to some function $f$. Multiplying by $\varepsilon$ the weak formulation of (1) written with the test function $\eta(t) \varphi(x, p), \eta \in C_{c}^{1}\left(\mathbb{R}_{+}\right), \varphi \in C_{c}^{1}\left(\mathbb{R}^{3} \times \mathbb{R}^{3}\right)$ and passing to the limit for $\varepsilon \searrow 0$ imply that $f(t) \in \operatorname{ker} \mathcal{T}, t \in \mathbb{R}_{+}$. Therefore there is a function $g=g(t, x, r, z)$ such that

$$
f(t, x, p)=g(t, x,|p \wedge b(x)|,(p \cdot b(x))), \quad(t, x, p) \in \mathbb{R}_{+} \times \mathbb{R}^{3} \times \mathbb{R}^{3} .
$$


Choosing now smooth test functions $\varphi$ which belong to the kernel of $\mathcal{T}$ yields by letting $\varepsilon \searrow 0(\operatorname{see}(32))$

$$
\begin{aligned}
& -\int_{\mathbb{R}_{+}} \eta^{\prime}(t) \int_{\mathbb{R}^{3}} \int_{\mathbb{R}^{3}} f(t, x, p) \varphi(x, p) \mathrm{d} p \mathrm{~d} x \mathrm{~d} t-\eta(0) \int_{\mathbb{R}^{3}} \int_{\mathbb{R}^{3}} f^{\mathrm{in}}(x, p) \varphi(x, p) \mathrm{d} p \mathrm{~d} x \\
& -\int_{\mathbb{R}_{+}} \eta(t) \int_{\mathbb{R}^{3}} \int_{\mathbb{R}^{3}} f(t, x, p)\left(\frac{p}{m} \cdot \nabla_{x} \varphi+q E(t, x) \cdot \nabla_{p} \varphi\right) \mathrm{d} p \mathrm{~d} x \mathrm{~d} t=0 .
\end{aligned}
$$

We are done if we prove that the formulation (39) is equivalent to the transport problem (31), (33). For doing this we transform all the integrals in (39) by using the symmetries of $f$ cf. (38) and the properties of the average operator. Since $\varphi$ belongs to the kernel of $\mathcal{T}$, there is a function $\psi$ such that $\varphi(x, p)=\psi(x,|p \wedge b(x)|,(p \cdot b(x))),(x, p) \in \mathbb{R}^{3} \times \mathbb{R}^{3}$. It is easily seen, by using cylindrical coordinates along the magnetic axis, that

$$
-\int_{\mathbb{R}_{+}} \eta^{\prime}(t) \int_{\mathbb{R}^{3}} \int_{\mathbb{R}^{3}} f \varphi \mathrm{d} p \mathrm{~d} x \mathrm{~d} t=-\int_{\mathbb{R}_{+}} \int_{\mathbb{R}^{3}} \int_{\mathbb{R}_{+}} \int_{\mathbb{R}} g(t, x, r, z) \partial_{t}(\eta \psi 2 \pi r) \mathrm{d} z \mathrm{~d} r \mathrm{~d} x \mathrm{~d} t
$$

and

$$
\begin{aligned}
-\eta(0) \int_{\mathbb{R}^{3}} \int_{\mathbb{R}^{3}} f^{\text {in }} \varphi \mathrm{d} p \mathrm{~d} x & =-\eta(0) \int_{\mathbb{R}^{3}} \int_{\mathbb{R}^{3}}\left\langle f^{\mathrm{in}}\right\rangle \varphi \mathrm{d} p \mathrm{~d} x \\
& =-\int_{\mathbb{R}^{3}} \int_{\mathbb{R}_{+}} \int_{\mathbb{R}} g^{\mathrm{in}}(x, r, z)(\eta(0) \psi 2 \pi r) \mathrm{d} z \mathrm{~d} r \mathrm{~d} x .
\end{aligned}
$$

For the last integral in (39) we appeal to Lemmas 3.2, 3.3.

$$
\begin{aligned}
& -\int_{\mathbb{R}_{+}} \eta(t) \int_{\mathbb{R}^{3}} \int_{\mathbb{R}^{3}} f(t, x, p)\left(\frac{p}{m} \cdot \nabla_{x} \varphi+q E(t, x) \cdot \nabla_{p} \varphi\right) \mathrm{d} p \mathrm{~d} x \mathrm{~d} t= \\
& -\int_{\mathbb{R}_{+}} \eta(t) \int_{\mathbb{R}^{3}} \int_{\mathbb{R}^{3}} f(t, x, p)\left\langle\frac{p}{m} \cdot \nabla_{x} \varphi+q E(t, x) \cdot \nabla_{p} \varphi\right\rangle \mathrm{d} p \mathrm{~d} x \mathrm{~d} t= \\
& -\int_{\mathbb{R}_{+}} \eta(t) \int_{\mathbb{R}^{3}} \int_{\mathbb{R}^{3}} f\left\{b(x) \otimes b(x) \frac{p}{m} \cdot \nabla_{x} \psi-\frac{(p \cdot b(x))|p \wedge b(x)|}{2 m} \operatorname{div}_{x} b \partial_{r} \psi\right. \\
& \left.+\frac{|p \wedge b(x)|^{2}}{2 m} \operatorname{div}_{x} b \partial_{z} \psi+q(E(t, x) \cdot b(x)) \partial_{z} \psi\right\} \mathrm{d} p \mathrm{~d} x \mathrm{~d} t= \\
& -\int_{\mathbb{R}_{+}} \int_{\mathbb{R}^{3}} \int_{\mathbb{R}_{+}} \int_{\mathbb{R}^{2}} g(t, x, r, z)\left\{\operatorname{div}_{x}\left(\eta \psi 2 \pi r \frac{z}{m} b(x)\right)-\partial_{r}\left(\eta \psi 2 \pi r \frac{z r}{2 m} \operatorname{div}_{x} b\right)\right. \\
& \left.+\partial_{z}\left(\eta \psi 2 \pi r\left(\frac{r^{2}}{2 m} \operatorname{div}_{x} b+q E(t, x) \cdot b(x)\right)\right)\right\} \mathrm{d} z \mathrm{~d} r \mathrm{~d} x \mathrm{~d} t .
\end{aligned}
$$

Gathering together (40), (41), (42) in (39) yields exactly the weak formulation of (26), (33) and therefore $f$ solves (31), (33). By the uniqueness of the solution of (31), (33) we deduce that all the family $\left(f^{\varepsilon}\right)_{\varepsilon>0}$ converges weakly $\star$ in $L^{\infty}\left(\mathbb{R}_{+} ; L^{2}\left(\mathbb{R}^{3} \times \mathbb{R}^{3}\right)\right)$ towards $f$. 
We inquire now about the strong convergence of the family $\left(f^{\varepsilon}\right)_{\varepsilon>0}$ when the initial conditions are well prepared

$$
\lim _{\varepsilon \backslash 0} f_{\varepsilon}^{\text {in }}=f^{\text {in }} \in \operatorname{ker} \mathcal{T} \text { strongly in } L^{2}\left(\mathbb{R}^{3} \times \mathbb{R}^{3}\right) .
$$

These results are definitely much difficult to establish and require smoothness for the solution of the limit model. The key point is the characterization for the solvability of $\mathcal{T} u=v($ see Proposition 2.2): $v \in$ Range $\mathcal{T}$ iff $\langle v\rangle=0$.

Theorem 4.2 Assume that $\left(f_{\varepsilon}^{\text {in }}\right)_{\varepsilon>0}$ are smooth and converge strongly in $L^{2}\left(\mathbb{R}^{3} \times \mathbb{R}^{3}\right)$ as $\varepsilon \searrow 0$ towards some function $f^{\text {in }} \in \operatorname{ker} \mathcal{T} \cap C_{c}^{2}\left(\mathbb{R}^{3} \times \mathbb{R}^{3}\right)$ verifying

$$
f^{\text {in }}(x, p)=0, \quad(x, p) \in \mathbb{R}^{3} \times \mathbb{R}^{3}, \quad|p \wedge b(x)| \leq r^{\text {in }}
$$

for some $r^{\text {in }}>0$. We suppose that $E \in L_{\text {loc }}^{\infty}\left(\mathbb{R}_{+} ; W^{1, \infty}\left(\mathbb{R}^{3}\right)\right)^{3}, \partial_{t} E \in L_{\text {loc }}^{\infty}\left(\mathbb{R}_{+} ; L^{\infty}\left(\mathbb{R}^{3}\right)\right)^{3}$, $b \in W^{2, \infty}\left(\mathbb{R}^{3}\right)^{3}, B \in W^{1, \infty}\left(\mathbb{R}^{3}\right), \operatorname{div}_{x}(B b)=0$ and $\inf _{x \in \mathbb{R}^{3}} B(x)>0$. Then $\left(f^{\varepsilon}\right)_{\varepsilon>0}$ converges strongly in $L_{\mathrm{loc}}^{\infty}\left(\mathbb{R}_{+} ; L^{2}\left(\mathbb{R}^{3} \times \mathbb{R}^{3}\right)\right)$ as $\varepsilon \searrow 0$ to $f$. In particular if $\left(\left(f_{\varepsilon}^{\text {in }}-f^{\text {in }}\right) / \varepsilon\right)_{\varepsilon>0}$ is bounded in $L^{2}\left(\mathbb{R}^{3} \times \mathbb{R}^{3}\right)$, then $\left(\left(f^{\varepsilon}-f\right) / \varepsilon\right)_{\varepsilon>0}$ is bounded in $L_{\mathrm{loc}}^{\infty}\left(\mathbb{R}_{+} ; L^{2}\left(\mathbb{R}^{3} \times \mathbb{R}^{3}\right)\right)$.

Proof. Notice that the characteristics $(X, P)$ in (34) satisfies

$$
\begin{gathered}
|X(t)-X(0)| \leq \int_{0}^{t} \frac{|P(s)|}{m} \mathrm{~d} s \\
|| P(t)|-| P(0)|| \leq|q| \int_{0}^{t}\|E(s)\|_{L^{\infty}\left(\mathbb{R}^{3}\right)} \mathrm{d} s
\end{gathered}
$$

and therefore it is easily seen that at any time $t \in \mathbb{R}_{+}$the solution $f$ of (31), (33) has compact support. Indeed, if

$$
\operatorname{supp} f^{\text {in }} \subset\left\{(x, p) \in \mathbb{R}^{3} \times \mathbb{R}^{3}:|x| \leq L_{x},|p| \leq L_{p}\right\}
$$

then for any $t \in[0, T], T \in \mathbb{R}_{+}$we have

$$
\operatorname{supp} f(t, \cdot, \cdot) \subset\left\{(x, p) \in \mathbb{R}^{3} \times \mathbb{R}^{3}:|x| \leq L_{x}^{T},|p| \leq L_{p}^{T}\right\}
$$

where

$$
L_{p}^{T}=L_{p}+|q| \int_{0}^{T}\|E(s)\|_{L^{\infty}\left(\mathbb{R}^{3}\right)} \mathrm{d} s, \quad L_{x}^{T}=L_{x}+\frac{T}{m} L_{p}^{T} .
$$

Moreover, the regularity of the electro-magnetic field guarantees that for any $T \in \mathbb{R}_{+}$

$$
\nabla_{t, x, p}^{2}(X, P)(s ; t, \cdot, \cdot) \in L^{\infty}\left(\mathbb{R}^{3} \times \mathbb{R}^{3}\right)
$$


uniformly with respect to $s, t \in[0, T]$ and therefore the strong solution $f$ of $(31),(33)$, which is given by

$$
f(t, x, p)=f^{\text {in }}((X, P)(0 ; t, x, p))
$$

belongs to $W^{2, \infty}\left([0, T] \times \mathbb{R}^{3} \times \mathbb{R}^{3}\right)$. In particular, the compactness of the support yields

$$
\begin{gathered}
\partial_{t} f+\frac{p}{m} \cdot \nabla_{x} f+q E(t, x) \cdot \nabla_{p} f \in L^{\infty}\left([0, T] ; W^{1,1} \cap W^{1, \infty}\left(\mathbb{R}^{3} \times \mathbb{R}^{3}\right)\right) \\
\partial_{t}\left(\partial_{t} f+\frac{p}{m} \cdot \nabla_{x} f+q E(t, x) \cdot \nabla_{p} f\right) \in L^{\infty}\left([0, T] ; L^{2}\left(\mathbb{R}^{3} \times \mathbb{R}^{3}\right)\right) .
\end{gathered}
$$

The solution $f$ of the limit model (31), (33) satisfies

$$
f(0)=f^{\text {in }},\left\langle\partial_{t} f+\frac{p}{m} \cdot \nabla_{x} f+q E(t, x) \cdot \nabla_{p} f\right\rangle=0, \quad \mathcal{T} f(t)=0, \quad t \in \mathbb{R}_{+} .
$$

By Proposition 2.2 there is a unique function $h$ such that

$$
h(t) \in \mathrm{D}(\mathcal{T}), \quad \partial_{t} f+\frac{p}{m} \cdot \nabla_{x} f+q E(t, x) \cdot \nabla_{p} f+\mathcal{T} h(t)=0, \quad\langle h(t)\rangle=0, \quad t \in \mathbb{R}_{+} .
$$

By Remark 2.1, Corollary 2.1 and Proposition 5.10 the corrector function $h(t, \cdot, \cdot)$ has compact support in $\mathbb{R}^{3} \times \mathbb{R}^{3}$, uniformly with respect to $t \in[0, T]$, and the regularity

$$
h \in L^{\infty}\left([0, T] ; W^{1,1} \cap W^{1, \infty}\left(\mathbb{R}^{3} \times \mathbb{R}^{3}\right)\right), \quad \partial_{t} h \in L^{\infty}\left([0, T] ; L^{2}\left(\mathbb{R}^{3} \times \mathbb{R}^{3}\right)\right) .
$$

We deduce that

$$
\partial_{t} h+\frac{p}{m} \cdot \nabla_{x} h+q E(t, x) \cdot \nabla_{p} h \in L^{\infty}\left([0, T] ; L^{2}\left(\mathbb{R}^{3} \times \mathbb{R}^{3}\right)\right)
$$

and thus combining (1), (43) and $\mathcal{T} f=0$ yields

$$
\begin{aligned}
& \partial_{t}\left(f^{\varepsilon}-f-\varepsilon h\right)+\frac{p}{m} \cdot \nabla_{x}\left(f^{\varepsilon}-f-\varepsilon h\right)+q E(t) \cdot \nabla_{p}\left(f^{\varepsilon}-f-\varepsilon h\right)+\frac{1}{\varepsilon} \mathcal{T}\left(f^{\varepsilon}-f-\varepsilon h\right)= \\
- & \varepsilon\left(\partial_{t} h+\frac{p}{m} \cdot \nabla_{x} h+q E(t) \cdot \nabla_{p} h\right) .
\end{aligned}
$$

Multiplying by $f^{\varepsilon}-f-\varepsilon h$ and integrating over $\mathbb{R}^{3} \times \mathbb{R}^{3}$ one gets

$$
\frac{1}{2} \frac{\mathrm{d}}{\mathrm{d} t}\left\|f^{\varepsilon}-f-\varepsilon h\right\|^{2} \leq \varepsilon\left\|\partial_{t} h+\frac{p}{m} \cdot \nabla_{x} h+q E(t) \cdot \nabla_{p} h\right\|\left\|f^{\varepsilon}-f-\varepsilon h\right\|
$$

implying that

$\left\|\left(f^{\varepsilon}-f-\varepsilon h\right)(t)\right\| \leq\left\|f_{\varepsilon}^{\text {in }}-f^{\text {in }}-\varepsilon h(0)\right\|+\varepsilon \int_{0}^{t}\left\|\partial_{t} h+\frac{p}{m} \cdot \nabla_{x} h+q E(s) \cdot \nabla_{p} h\right\| \mathrm{d} s, \quad t \in \mathbb{R}_{+}$.

Therefore for any $T>0$ there is a constant $C_{T}$ not depending on $\varepsilon>0$ such that

$$
\left\|f^{\varepsilon}(t)-f(t)\right\| \leq\left\|f_{\varepsilon}^{\text {in }}-f^{\text {in }}\right\|+C_{T} \varepsilon, \quad t \in[0, T], \varepsilon>0
$$

and our conclusions follow immediately. 


\section{Second order gyrokinetic Vlasov equation}

In the previous section it was shown that up to $\mathcal{O}(\varepsilon)$ terms, the particle distributions $\left(f^{\varepsilon}\right)_{\varepsilon>0}$ solving (1), (2) behave like the solution of the limit model (31) (or equivalently (26)) (33).

The main motivation of the guiding-center approximation relies on the confinement properties. We have seen that at the lowest order the particles are advected along the magnetic lines and therefore the plasma remains confined provided that the magnetic field shape is such that the magnetic lines are closed into a bounded domain. Nevertheless, in order to approximate the confinement time we need to compute the drift velocities in the orthogonal directions to the magnetic lines corresponding to the first order correction $\left(X^{1}, P^{1}\right)$ of the particle dynamics $\left(X^{\varepsilon}, P^{\varepsilon}\right)$ in $(6)$. We inquire about higher order approximation for the Vlasov equation with large magnetic field. Certainly we may expect that the second order approximation follows by similar arguments. Actually this analysis requires much more computations. In this case is convenient to establish first some general results on abstract average operators. It mainly concerns their commutation properties with respect to first order differential operators. Next we will appeal to these results and we will obtain the second order approximation for the Vlasov equation (1).

\subsection{Average operator revisited}

In this section the notation $b^{0}$ stands for a given field $b^{0}: \mathbb{R}^{m} \rightarrow \mathbb{R}^{m}$ satisfying

$$
\begin{gathered}
b^{0} \in W_{\mathrm{loc}}^{1, \infty}\left(\mathbb{R}^{m}\right) \\
\operatorname{div}_{y} b^{0}=0
\end{gathered}
$$

and the growth condition

$$
\exists C>0:\left|b^{0}(y)\right| \leq C(1+|y|), \quad y \in \mathbb{R}^{m}
$$

Under the above hypotheses the characteristic flow $Y=Y(s ; y)$ is well defined

$$
\begin{gathered}
\frac{d Y}{d s}=b^{0}(Y(s ; y)), \quad(s, y) \in \mathbb{R} \times \mathbb{R}^{m} \\
Y(0 ; y)=y, \quad y \in \mathbb{R}^{m}
\end{gathered}
$$


and has the regularity $Y \in W_{\text {loc }}^{1, \infty}\left(\mathbb{R} \times \mathbb{R}^{m}\right)$. By (45) we deduce that for any $s \in \mathbb{R}$, the map $y \rightarrow Y(s ; y)$ is measure preserving

$$
\int_{\mathbb{R}^{m}} \theta(Y(s ; y)) \mathrm{d} y=\int_{\mathbb{R}^{m}} \theta(y) \mathrm{d} y, \quad \forall \theta \in L^{1}\left(\mathbb{R}^{m}\right) .
$$

We have the following standard result concerning the kernel of $u \rightarrow \mathcal{T} u=\operatorname{div}_{y}\left(b^{0}(y) u(y)\right)$.

Proposition 5.1 Let $u \in L_{\text {loc }}^{1}\left(\mathbb{R}^{m}\right)$. Then $\operatorname{div}_{y}\left(b^{0}(y) u(y)\right)=0$ in $\mathcal{D}^{\prime}\left(\mathbb{R}^{m}\right)$ iff for any $s \in \mathbb{R}$ we have $u(Y(s ; y))=u(y)$ for a.a. $y \in \mathbb{R}^{m}$.

We denote by $T$ the linear operator defined by $T u=\operatorname{div}_{y}\left(b^{0}(y) u(y)\right)$ for any $u$ in the domain

$$
\mathrm{D}(T)=\left\{u \in L^{2}\left(\mathbb{R}^{m}\right): \operatorname{div}_{y}\left(b^{0}(y) u(y)\right) \in L^{2}\left(\mathbb{R}^{m}\right)\right\}
$$

Thanks to Proposition 5.1 we have

$$
\operatorname{ker} T=\left\{u \in L^{2}\left(\mathbb{R}^{m}\right): u(Y(s ; y))=u(y), s \in \mathbb{R}, \text { a.e. } y \in \mathbb{R}^{m}\right\}
$$

Following the ideas in [4] we introduce the average operator along the measure preserving flow $Y$.

Proposition 5.2 For any function $u \in L^{2}\left(\mathbb{R}^{m}\right)$ the averages $\left(T^{-1} \int_{0}^{T} u(Y(s ; \cdot)) \mathrm{d} s\right)_{T>0}$, $\left(T^{-1} \int_{-T}^{0} u(Y(s ; \cdot)) \mathrm{d} s\right)_{T>0}$ converge strongly in $L^{2}\left(\mathbb{R}^{m}\right)$ as $T \rightarrow+\infty$ towards some function denoted $\langle u\rangle \in \operatorname{ker} T$. The average operator $u \rightarrow\langle u\rangle$ is linear continuous on $L^{2}\left(\mathbb{R}^{m}\right)$. Moreover it coincides with the orthogonal projection on the kernel of $T$ i.e.,

$$
\langle u\rangle \in \operatorname{ker} T: \int_{\mathbb{R}^{m}}(u-\langle u\rangle) \varphi(y) \mathrm{d} y=0, \quad \varphi \in \operatorname{ker} T .
$$

As in Section 2 we have

$$
\operatorname{ker}\langle\cdot\rangle=\operatorname{ker} \operatorname{Proj} j_{\operatorname{ker} T}={ }^{\perp}(\operatorname{ker} T)={ }^{\perp}\left(\operatorname{ker} T^{\star}\right)=\overline{\operatorname{Range} T}
$$

and for any $u \in L^{2}\left(\mathbb{R}^{m}\right)$ we have the orthogonal decomposition

$$
u=(u-\langle u\rangle)+\langle u\rangle, \quad \int_{\mathbb{R}^{m}}(u-\langle u\rangle)\langle u\rangle \mathrm{d} y=0 .
$$

We will assume that the range of $T$ is closed. This is the case for strongly magnetized plasmas, cf. Proposition 2.2 i.e.,

$$
\text { Range } T=\operatorname{ker}\langle\cdot\rangle \text {. }
$$


We are looking now for first order differential operators commuting with the average operator. Consider a smooth field $c$ with bounded divergence

$$
c \in W_{\mathrm{loc}}^{1, \infty}\left(\mathbb{R}^{m}\right), \quad \operatorname{div}_{y} c \in L^{\infty}\left(\mathbb{R}^{m}\right)
$$

and let us denote by $Z$ the flow associated to $c$ (we assume that $Z$ is well defined for any $\left.(s, y) \in \mathbb{R} \times \mathbb{R}^{m}\right)$. We consider the operator $c \cdot \nabla_{y}$

$\mathrm{D}\left(c \cdot \nabla_{y}\right)=\left\{u \in L^{2}\left(\mathbb{R}^{m}\right): \operatorname{div}_{y}(c u) \in L^{2}\left(\mathbb{R}^{m}\right)\right\}, c \cdot \nabla_{y} u=\operatorname{div}_{y}(c u)-\left(\operatorname{div}_{y} c\right) u, \quad u \in \mathrm{D}\left(c \cdot \nabla_{y}\right)$

where the divergence is understood in distribution sense i.e., there is a function $v \in$ $L^{2}\left(\mathbb{R}^{m}\right)$ such that

$$
\int_{\mathbb{R}^{m}} v \varphi \mathrm{d} y+\int_{\mathbb{R}^{m}} u\left(c \cdot \nabla_{y} \varphi\right) \mathrm{d} y=0
$$

for any function $\varphi \in C_{c}^{1}\left(\mathbb{R}^{m}\right)$ or equivalently

$$
\sup _{\varphi \neq 0, \varphi \in C_{c}^{1}\left(\mathbb{R}^{m}\right)} \frac{\left|\int_{\mathbb{R}^{m}} u\left(c \cdot \nabla_{y} \varphi\right) \mathrm{d} y\right|}{\|\varphi\|_{L^{2}\left(\mathbb{R}^{m}\right)}}<+\infty .
$$

We want to determine the fields $c$ such that $c \cdot \nabla_{y}$ is commuting with $\langle\cdot\rangle$ i.e., for any $u \in \mathrm{D}\left(c \cdot \nabla_{y}\right)$ the average $\langle u\rangle$ belongs to $\mathrm{D}\left(c \cdot \nabla_{y}\right)$ and

$$
c \cdot \nabla_{y}\langle u\rangle=\left\langle c \cdot \nabla_{y} u\right\rangle .
$$

Notice that the differential operator associated to such a field $c$ leaves invariant the kernel of $T$

$$
\forall u \in \mathrm{D}\left(c \cdot \nabla_{y}\right) \cap \operatorname{ker} T, \quad c \cdot \nabla_{y} u \in \operatorname{ker} T .
$$

Indeed, for any $u \in \mathrm{D}\left(c \cdot \nabla_{y}\right) \cap \operatorname{ker} T$ we have $u=\langle u\rangle$ and

$$
c \cdot \nabla_{y} u=c \cdot \nabla_{y}\langle u\rangle=\left\langle c \cdot \nabla_{y} u\right\rangle \in \operatorname{ker} T \text {. }
$$

Actually the condition (50) can be written in a simpler form. For any function $u \in$ $L^{2}\left(\mathbb{R}^{m}\right)$ the notation $u_{h}$ stands for the translation $u(Z(h ; \cdot))$. Since $\operatorname{div}_{y} c$ is bounded, notice that $u_{h} \in L^{2}\left(\mathbb{R}^{m}\right)$ for any $h \in \mathbb{R}$. We appeal to the standard result (see [8], Proposition IX.3, pp. 153 for similar results).

Lemma 5.1 Let $u$ be a function in $L^{2}\left(\mathbb{R}^{m}\right)$. Then the following statements are equivalent 
a) $u \in \mathrm{D}\left(c \cdot \nabla_{y}\right)$.

b) $\left(h^{-1}\left(u_{h}-u\right)\right)_{h}$ is bounded in $L^{2}\left(\mathbb{R}^{m}\right)$.

Moreover, for any $u \in \mathrm{D}\left(c \cdot \nabla_{y}\right)$ we have the convergence

$$
\lim _{h \rightarrow 0} \frac{u_{h}-u}{h}=c \cdot \nabla_{y} u, \text { strongly in } L^{2}\left(\mathbb{R}^{m}\right) .
$$

We have the following formula of integration by parts.

Lemma 5.2 For any function $u, \varphi \in \mathrm{D}\left(c \cdot \nabla_{y}\right)$ we have

$$
\int_{\mathbb{R}^{m}}\left(c \cdot \nabla_{y} \varphi\right) u \mathrm{~d} y+\int_{\mathbb{R}^{m}}\left(c \cdot \nabla_{y} u\right) \varphi \mathrm{d} y+\int_{\mathbb{R}^{m}}\left(\operatorname{div}_{y} c\right) u \varphi \mathrm{d} y=0 .
$$

Based on the characterization in Lemma 5.1 we prove that (50) is equivalent to the invariance of $\operatorname{ker} T$ by the translations $u \rightarrow u_{h}$.

Proposition 5.3 A smooth field c with bounded divergence satisfies (50) iff the translations parallel to c leave invariant the kernel of $T$ i.e.,

$$
\forall h \in \mathbb{R}, \forall u \in \operatorname{ker} T \text { then } u_{h} \in \operatorname{ker} T \text {. }
$$

Proof. Assume that (51) holds true and let us consider $u \in \mathrm{D}\left(c \cdot \nabla_{y}\right) \cap \operatorname{ker} T$. By Lemma 5.1 we know that

$$
c \cdot \nabla_{y} u=\lim _{h \rightarrow 0} \frac{u_{h}-u}{h} \text { strongly in } L^{2}\left(\mathbb{R}^{m}\right) .
$$

But for any $h \neq 0$ we have $\left(u_{h}-u\right) / h \in \operatorname{ker} T$ and since $\operatorname{ker} T$ is closed (because $T$ is closed) we deduce that $c \cdot \nabla_{y} u \in \operatorname{ker} T$.

Assume now that (50) holds true and let us establish (51). For the sake of simplicity we assume that the field $b^{0}$ possesses a complete family of smooth independent prime integrals denoted $\psi_{1}, \ldots, \psi_{m-1}$ i.e.,

$$
b^{0} \cdot \nabla_{y} \psi_{i}=0, \quad i \in\{1,2, \ldots, m-1\}, \quad \operatorname{rank} \partial_{y} \psi(y)=m-1, \quad y \in \mathbb{R}^{m}
$$

where $\psi={ }^{t}\left(\psi_{1}, \ldots, \psi_{m-1}\right)$. Actually this is enough for our purpose since the above hypotheses hold true for strongly magnetized plasmas. Notice that it is sufficient to consider $u=\psi_{i}, i \in\{1,2, \ldots, m-1\}$. We can write

$$
\frac{\mathrm{d}}{\mathrm{d} h} \psi_{i}(Z(h ; y))=\left(c \cdot \nabla_{y} \psi_{i}\right)(Z(h ; y))
$$


But $c \cdot \nabla_{y} \psi_{i} \in \operatorname{ker} T$ and therefore

$$
c \cdot \nabla_{y} \psi_{i}=v_{i}\left(\psi_{1}(y), \ldots, \psi_{m-1}(y)\right), \quad y \in \mathbb{R}^{m}
$$

for some smooth function $v_{i}$ implying that

$$
\frac{\mathrm{d}}{\mathrm{d} h} \psi_{i}(Z(h ; y))=v_{i}\left(\psi_{1}(Z(h ; y)), \ldots, \psi_{m-1}(Z(h ; y))\right), \quad y \in \mathbb{R}^{m} .
$$

Similarly we have

$$
\frac{\mathrm{d}}{\mathrm{d} h} \psi_{i}(Z(h ; Y(s ; y)))=v_{i}\left(\psi_{1}(Z(h ; Y(s ; y))), \ldots, \psi_{m-1}(Z(h ; Y(s ; y)))\right), \quad y \in \mathbb{R}^{m} .
$$

The functions $h \rightarrow \psi(Z(h ; y))$ and $h \rightarrow \psi(Z(h ; Y(s ; y)))$ satisfy the same system of differential equations and the same initial conditions

$$
\psi(Z(0 ; y))=\psi(y)=\psi(Y(s ; y))=\psi(Z(0 ; Y(s ; y)))
$$

By the uniqueness of the solution we deduce that

$$
\psi(Z(h ; Y(s ; y)))=\psi(Z(h ; y)), h, s \in \mathbb{R}
$$

saying that $\psi_{h}$ is constant along the flow $Y$.

Remark 5.1 In the sequel we will need to pick test functions $\varphi \in \mathrm{D}\left(c \cdot \nabla_{y}\right) \cap \operatorname{ker} T$. When the field $b^{0}$ possesses a complete family of smooth independent prime integrals $\psi_{1}, \ldots, \psi_{m-1}$ verifying

$$
\lim _{|y| \rightarrow+\infty}\left(\left|\psi_{1}(y)\right|+\ldots+\left|\psi_{m-1}(y)\right|\right)=+\infty
$$

it is easily seen that for any function $v \in C_{c}^{1}\left(\mathbb{R}^{m-1}\right)$ the function $y \rightarrow v\left(\psi_{1}(y), \ldots, \psi_{m-1}(y)\right)$ belongs to $C_{c}^{1}\left(\mathbb{R}^{m}\right) \cap \operatorname{ker} T$ which is contained in $\mathrm{D}\left(c \cdot \nabla_{y}\right) \cap \operatorname{ker} T$.

Adapting the arguments in the proof of Lemma 5.1 yields a similar characterization for the elements of $\mathrm{D}\left(c \cdot \nabla_{y}\right) \cap \operatorname{ker} T$.

Proposition 5.4 Consider a smooth field $c=c(y)$ with bounded divergence such that $c \cdot \nabla_{y}$ leaves invariant the kernel of $T$ and a function $u \in \operatorname{ker} T$. Then $u$ belongs to $\mathrm{D}\left(c \cdot \nabla_{y}\right)$ iff

$$
\sup _{\varphi \neq 0, \varphi \in C_{c}^{1}\left(\mathbb{R}^{m}\right) \cap \operatorname{ker} T} \frac{\left|\int_{\mathbb{R}^{m}}\left(c \cdot \nabla_{y} \varphi\right) u \mathrm{~d} y\right|}{\|\varphi\|_{L^{2}\left(\mathbb{R}^{m}\right)}}<+\infty .
$$


Proof. We only indicate the main lines. The key point is that for functions $u \in \operatorname{ker} T$ the uniform bound in (53) (when $\varphi$ spans only the constant functions along the flow $Y$ ) ensures the boundedness of $\left(\frac{u_{h}-u}{h}\right)_{h}$ in $L^{2}\left(\mathbb{R}^{m}\right)$, which implies, by Lemma 5.1, that $u \in \mathrm{D}\left(c \cdot \nabla_{y}\right)$. Indeed, since by Proposition 5.3 we know that the translation parallel to $c$ leaves invariant $\operatorname{ker} T$, when estimating the $L^{2}\left(\mathbb{R}^{m}\right)$ norm of $\frac{u_{h}-u}{h}$ it is sufficient to integrate against smooth test functions $\varphi \in C_{c}^{1}\left(\mathbb{R}^{m}\right) \cap \operatorname{ker} T$. After standard computations we obtain a uniform bound for the $L^{2}$ norms of $\left(\frac{u_{h}-u}{h}\right)_{h}$ provided that (53) holds true.

We are ready now to establish the following result for differential operators leaving invariant the kernel of $T$.

Proposition 5.5 Consider a smooth field $c=c(y)$ with bounded divergence such that the operator $c \cdot \nabla_{y}$ leaves invariant the kernel of $T$. Then for any function $u \in \mathrm{D}\left(c \cdot \nabla_{y}\right)$ the average $\langle u\rangle$ belongs to $\mathrm{D}\left(c \cdot \nabla_{y}\right)$ and

$$
c \cdot \nabla_{y}\langle u\rangle-\left\langle c \cdot \nabla_{y} u\right\rangle=\left\langle(u-\langle u\rangle) \operatorname{div}_{y} c\right\rangle .
$$

Proof. Let us consider $u \in \mathrm{D}\left(c \cdot \nabla_{y}\right)$. Using the variational charaterization of the average operator and the integration by parts formula in Lemma 5.2 we can write for any test function $\varphi \in C_{c}^{1}\left(\mathbb{R}^{m}\right) \cap \operatorname{ker} T$

$$
\begin{aligned}
\int_{\mathbb{R}^{m}}\left\langle c \cdot \nabla_{y} u\right\rangle \varphi \mathrm{d} y & =\int_{\mathbb{R}^{m}}\left(c \cdot \nabla_{y} u\right) \varphi \mathrm{d} y \\
& =-\int_{\mathbb{R}^{m}} u \varphi \operatorname{div}_{y} c \mathrm{~d} y-\int_{\mathbb{R}^{m}}\left(c \cdot \nabla_{y} \varphi\right) u \mathrm{~d} y .
\end{aligned}
$$

Since $c \cdot \nabla_{y}$ leaves invariant the kernel of $T$, the function $c \cdot \nabla_{y} \varphi$ belongs to $\operatorname{ker} T$. We have

$$
\int_{\mathbb{R}^{m}}\left(c \cdot \nabla_{y} \varphi\right) u \mathrm{~d} y=\int_{\mathbb{R}^{m}}\left(c \cdot \nabla_{y} \varphi\right)\langle u\rangle \mathrm{d} y
$$

and therefore

$$
\int_{\mathbb{R}^{m}}\left(c \cdot \nabla_{y} \varphi\right)\langle u\rangle \mathrm{d} y=-\int_{\mathbb{R}^{m}}\left(u \operatorname{div}_{y} c+c \cdot \nabla_{y} u\right) \varphi \mathrm{d} y
$$

implying that

$$
\sup _{\varphi \neq 0, \varphi \in C_{c}^{1}\left(\mathbb{R}^{m}\right) \cap \operatorname{ker} T} \frac{\left|\int_{\mathbb{R}^{m}}\left(c \cdot \nabla_{y} \varphi\right)\langle u\rangle \mathrm{d} y\right|}{\|\varphi\|_{L^{2}\left(\mathbb{R}^{m}\right)}} \leq\left\|\operatorname{div}_{y} c\right\|_{L^{\infty}\left(\mathbb{R}^{m}\right)}\|u\|_{L^{2}\left(\mathbb{R}^{m}\right)}+\left\|c \cdot \nabla_{y} u\right\|_{L^{2}\left(\mathbb{R}^{m}\right)} .
$$


By Proposition 5.4 we deduce that $\langle u\rangle \in \mathrm{D}\left(c \cdot \nabla_{y}\right)$ and coming back in (54) yields

$$
\begin{aligned}
\int_{\mathbb{R}^{m}}\left\langle c \cdot \nabla_{y} u\right\rangle \varphi \mathrm{d} y & =-\int_{\mathbb{R}^{m}} u \varphi \operatorname{div}_{y} c \mathrm{~d} y-\int_{\mathbb{R}^{m}}\left(c \cdot \nabla_{y} \varphi\right)\langle u\rangle \mathrm{d} y \\
& =\int_{\mathbb{R}^{m}}\left(c \cdot \nabla_{y}\langle u\rangle\right) \varphi \mathrm{d} y+\int_{\mathbb{R}^{m}}(\langle u\rangle-u) \varphi \operatorname{div}_{y} c \mathrm{~d} y \\
& =\int_{\mathbb{R}^{m}}\left(c \cdot \nabla_{y}\langle u\rangle\right) \varphi \mathrm{d} y+\int_{\mathbb{R}^{m}}\left\langle(\langle u\rangle-u) \operatorname{div}_{y} c\right\rangle \varphi \mathrm{d} y .
\end{aligned}
$$

Finally one gets for any $\varphi \in \operatorname{ker} T$

$$
\int_{\mathbb{R}^{m}}\left\{c \cdot \nabla_{y}\langle u\rangle-\left\langle c \cdot \nabla_{y} u\right\rangle-\left\langle(u-\langle u\rangle) \operatorname{div}_{y} c\right\rangle\right\} \varphi \mathrm{d} y=0
$$

and since $c \cdot \nabla_{y}\langle u\rangle \in \operatorname{ker} T$ (because $c \cdot \nabla_{y}$ leaves invariant the kernel of $T$ ) we deduce that

$$
c \cdot \nabla_{y}\langle u\rangle-\left\langle c \cdot \nabla_{y} u\right\rangle=\left\langle(u-\langle u\rangle) \operatorname{div}_{y} c\right\rangle .
$$

Remark 5.2 We may expect a simpler justification for Proposition 5.4 by extending the uniform bound in (53) to any smooth function in $L^{2}\left(\mathbb{R}^{m}\right)$, not necessarily in $\operatorname{ker} T$. Indeed for any $\varphi \in C_{c}^{1}\left(\mathbb{R}^{m}\right)$ we can use the orthogonal decomposition in $L^{2}\left(\mathbb{R}^{m}\right)$

$$
\varphi=\langle\varphi\rangle+(\varphi-\langle\varphi\rangle)
$$

then try to write for some constant $C$

$$
\left|\int_{\mathbb{R}^{m}}\left(c \cdot \nabla_{y}\langle\varphi\rangle\right) u \mathrm{~d} y\right| \leq C\|\langle\varphi\rangle\|_{L^{2}\left(\mathbb{R}^{m}\right)} \leq C\|\varphi\|_{L^{2}\left(\mathbb{R}^{m}\right)}
$$

(since $\langle\varphi\rangle \in \operatorname{ker} T$ ) by expecting that

$$
\left\langle c \cdot \nabla_{y}(\varphi-\langle\varphi\rangle)\right\rangle=0 \text {, which can be motivated by the fact that }\langle\varphi-\langle\varphi\rangle\rangle=0 \text {. }
$$

Actually (56) is not valid because it is not of all clear that $c \cdot \nabla_{y}$ leaves invariant the kernel of the average operator. Indeed, by Proposition 5.5 we have for any zero average function $\theta$

$$
\left\langle c \cdot \nabla_{y} \theta\right\rangle=-\left\langle\theta \operatorname{div}_{y} c\right\rangle
$$

which clearly says that the last assertion is false at least when $\operatorname{div}_{y} c$ is not constant along the flow $Y$. 
We formulate now necessary and sufficient conditions for fields to be commuting with the average operator.

Theorem 5.1 Consider a smooth field $c=c(y)$ with bounded divergence and assume that the field $b^{0}$ possesses a complete family of smooth independent prime integrals $\psi_{1}, \ldots, \psi_{m-1}$ verifying (52). Then the following statements are equivalent

i) the operators $c \cdot \nabla_{y}$ and $\langle\cdot\rangle$ are commuting;

ii) the operator $c \cdot \nabla_{y}$ leaves invariant the kernel of $T=b^{0} \cdot \nabla_{y}$ and $\operatorname{div}_{y} c$ is constant along the flow of $b^{0}$.

Proof. Assume that $c \cdot \nabla_{y}$ and $\langle\cdot\rangle$ are commuting. For any $u \in \mathrm{D}\left(c \cdot \nabla_{y}\right) \cap \operatorname{ker} T$ the average $\langle u\rangle=u$ belongs to $\mathrm{D}\left(c \cdot \nabla_{y}\right)$ and we have

$$
c \cdot \nabla_{y} u=c \cdot \nabla_{y}\langle u\rangle=\left\langle c \cdot \nabla_{y} u\right\rangle \in \operatorname{ker} T
$$

saying that $c \cdot \nabla_{y}$ leaves invariant the kernel of $T$. For any function $u \in \mathrm{D}\left(c \cdot \nabla_{y}\right)$ we know that $\langle u\rangle \in \mathrm{D}\left(c \cdot \nabla_{y}\right)$ and by Proposition 5.5 we deduce that

$$
0=c \cdot \nabla_{y}\langle u\rangle-\left\langle c \cdot \nabla_{y} u\right\rangle=\left\langle(u-\langle u\rangle) \operatorname{div}_{y} c\right\rangle
$$

Therefore for any $\varphi \in \operatorname{ker} T$ we have

$$
\int_{\mathbb{R}^{m}}(u-\langle u\rangle) \varphi \operatorname{div}_{y} c \mathrm{~d} y=0
$$

Let us consider $v \in C_{c}^{2}\left(\mathbb{R}^{m}\right)$ and take $u=T v \in C_{c}^{1}\left(\mathbb{R}^{m}\right) \subset \mathrm{D}\left(c \cdot \nabla_{y}\right)$ implying that

$$
\int_{\mathbb{R}^{m}} T v \varphi \operatorname{div}_{y} c \mathrm{~d} y=0, \quad \varphi \in \operatorname{ker} T
$$

We deduce that $\varphi \operatorname{div}_{y} c$ is constant along the flow of $b^{0}$ for any function $\varphi \in \operatorname{ker} T$. We are done if we are able to construct functions $\varphi \in \operatorname{ker} T$ with support including balls in $\mathbb{R}^{m}$ with radii as large as we want. For example, consider $w \in C_{c}^{1}\left(\mathbb{R}^{m-1}\right)$ such that $B(0,1) \subset \operatorname{supp} w$ and

$$
\varphi_{R}(y)=w\left(\frac{\psi_{1}(y)}{M}, \ldots, \frac{\psi_{m-1}(y)}{M}\right), \quad M=\sup _{|y| \leq R}\left(\left|\psi_{1}(y)\right|^{2}+\ldots+\left|\psi_{m-1}(y)\right|^{2}\right)^{1 / 2} .
$$

Clearly the condition (52) implies that for any $R>0, \varphi_{R} \in C_{c}^{1}\left(\mathbb{R}^{m}\right) \cap \operatorname{ker} T$ and for any $y \in B(0, R)$ we have $\varphi_{R}(y) \neq 0$ saying that $B(0, R) \subset \operatorname{supp} \varphi_{R}$. 
Conversely, assume that $c \cdot \nabla_{y}$ leaves invariant the kernel of $T$ and that $\operatorname{div}_{y} c$ is constant along the flow of $b^{0}$. By Proposition 5.5, for any $u \in \mathrm{D}\left(c \cdot \nabla_{y}\right)$ the average $\langle u\rangle$ belongs to $\mathrm{D}\left(c \cdot \nabla_{y}\right)$ and

$$
c \cdot \nabla_{y}\langle u\rangle-\left\langle c \cdot \nabla_{y} u\right\rangle=\left\langle(u-\langle u\rangle) \operatorname{div}_{y} c\right\rangle=\langle u-\langle u\rangle\rangle \operatorname{div}_{y} c=0 .
$$

For any $i \in\{1,2, \ldots, m-1\}$ consider the field $b^{i}$ orthogonal to $b^{0}$ and satisfying

$$
b^{i} \cdot \nabla_{y} \psi_{k}=\delta_{i k}, \quad k \in\{1, \ldots, m-1\} .
$$

Notice that the differential operators $b^{i} \cdot \nabla_{y}$ leave invariant the kernel of $T$ because for any, let say smooth, $u(y)=v\left(\psi_{1}(y), \ldots, \psi_{m-1}(y)\right) \in \operatorname{ker} T$ we have

$$
b^{i} \cdot \nabla_{y} u=\sum_{k=1}^{m-1} \frac{\partial v}{\partial \psi_{k}} b^{i} \cdot \nabla_{y} \psi_{k}=\frac{\partial v}{\partial \psi_{i}}(\psi(y)) \in \operatorname{ker} T .
$$

Therefore, by Proposition 5.5 we have for any $i \in\{1, \ldots, m-1\}$ and any $u \in \mathrm{D}\left(b^{i} \cdot \nabla_{y}\right)$

$$
\langle u\rangle \in \mathrm{D}\left(b^{i} \cdot \nabla_{y}\right), \quad b^{i} \cdot \nabla_{y}\langle u\rangle-\left\langle b^{i} \cdot \nabla_{y} u\right\rangle=\left\langle(u-\langle u\rangle) \operatorname{div}_{y} b^{i}\right\rangle .
$$

Obviously the previous statement also holds true for the operator $T=b^{0} \cdot \nabla_{y}$. Actually, for any $i \in\{1, \ldots, m-1\}$, it is possible to construct a first order differential operator $\tilde{b}^{i} \cdot \nabla_{y}$ commuting with the average operator. Indeed, consider $\tilde{b}^{i}=b^{i}-\lambda^{i} b^{0}$, where $T \lambda^{i}=\operatorname{div}_{y} b^{i}-\left\langle\operatorname{div}_{y} b^{i}\right\rangle,\left\langle\lambda^{i}\right\rangle=0$. It easily seen that $\tilde{b}^{i} \cdot \nabla_{y}$ leaves invariant the kernel of $T$ and that $\operatorname{div}_{y} \tilde{b}^{i}$ is constant along the flow of $b^{0}$

$$
\operatorname{div}_{y} \tilde{b}^{i}=\operatorname{div}_{y} b^{i}-T \lambda^{i}=\left\langle\operatorname{div}_{y} b^{i}\right\rangle
$$

Therefore Theorem 5.1 implies that $\tilde{b}^{i} \cdot \nabla_{y}$ and $\langle\cdot\rangle$ are commuting for any $i \in\{1, \ldots, m-$ $1\}$.

We compute now the fields $b^{i}, i \in\{1, \ldots, m-1\}$ in the specific case of strongly magnetized plasmas. We have $m=6, y=(x, p), b^{0}(x, p)=\left(0,0,0, \omega_{c}(x) p \wedge b(x)\right)$, $\mathcal{T}=\omega_{c}(x)(p \wedge b(x)) \cdot \nabla_{p}$

$\psi_{1}(x, p)=x_{1}, \quad \psi_{2}(x, p)=x_{2}, \quad \psi_{3}(x, p)=x_{3}, \quad \psi_{4}(x, p)=|p \wedge b(x)|, \quad \psi_{5}(x, p)=p \cdot b(x)$

and

$$
\langle u\rangle(x, p)=\frac{1}{2 \pi} \int_{S(x)} u(x,|p \wedge b(x)| \omega+(p \cdot b(x)) b(x)) \mathrm{d} \omega
$$


where $S(x)=\left\{\omega \in S^{2}: b(x) \cdot \omega=0\right\}$. Under the assumption $\inf _{x \in \mathbb{R}^{3}} B(x)>0$ we know by Proposition 2.2 that (49) holds true. Notice also that (52) is satisfied. We obtain the formula

$$
\begin{gathered}
\nabla_{x, p} \psi_{i}=\left(e_{i}, 0,0,0\right), i \in\{1,2,3\} \\
\nabla_{x, p} \psi_{4}=\left(-\frac{(p \cdot b(x))}{|p \wedge b(x)|}{ }^{t} \partial_{x} b p, \frac{p-(p \cdot b(x)) b}{|p \wedge b(x)|}\right), \quad \nabla_{x, p} \psi_{5}=\left({ }^{t} \partial_{x} b p, b\right) \\
b^{i}=\left(e_{i},-\frac{\left({ }^{t} \partial_{x} b p\right)_{i}}{|p \wedge b(x)|}{ }^{\perp} p\right), i \in\{1,2,3\}, b^{4}=\left(0,0,0, \frac{p-(p \cdot b(x)) b}{|p \wedge b(x)|}\right), b^{5}=(0,0,0, b)
\end{gathered}
$$

where ${ }^{\perp} p=|p \wedge b(x)| b-(p \cdot b(x)) \frac{p-(p \cdot b(x)) b}{|p \wedge b(x)|}$. Notice that

$$
\begin{gathered}
\operatorname{div}_{p}{ }^{\perp} p=-\frac{(p \cdot b(x))}{|p \wedge b(x)|}, \quad \operatorname{div}_{x, p} b^{i}=\frac{\left({ }^{t} \partial_{x} b p\right)_{i}}{|p \wedge b(x)|^{2}}(p \cdot b(x)), i \in\{1,2,3\} \\
\operatorname{div}_{x, p} b^{4}=\frac{1}{|p \wedge b(x)|}, \quad \operatorname{div}_{x, p} b^{5}=0
\end{gathered}
$$

\subsection{Multi-scale analysis}

The Vlasov equation describing the dynamics of strongly magnetized plasmas (1) is of the form

$$
\partial_{t} u^{\varepsilon}+a(t, y) \cdot \nabla_{y} u^{\varepsilon}+\frac{1}{\varepsilon} b^{0} \cdot \nabla_{y} u^{\varepsilon}=0, \quad t \in \mathbb{R}_{+}, \quad y \in \mathbb{R}^{m}
$$

with $m=6, y=(x, p), a(t, x, p)=(p / m, q E(t, x)), b^{0}(x, p)=\left(0,0,0, \omega_{c}(x) p \wedge b(x)\right)$. Notice that $\operatorname{div}_{x, p} a=\operatorname{div}_{x, p} b^{0}=0$. The ansatz $u^{\varepsilon}=u+\varepsilon u^{1}+\varepsilon^{2} u^{2}+\ldots$ leads to the sequence of equations

$$
\begin{gathered}
T u=b^{0} \cdot \nabla_{y} u=0 \\
\partial_{t} u+a(t, y) \cdot \nabla_{y} u+T u^{1}=0 \\
\partial_{t} u^{1}+a(t, y) \cdot \nabla_{y} u^{1}+T u^{2}=0
\end{gathered}
$$

The time evolution equation for the leading order term $u$ comes by applying the average operator to (63) and taking into account that $T u^{1} \in \operatorname{ker}\langle\cdot\rangle$. We obtain

$$
\partial_{t} u+\left\langle a(t) \cdot \nabla_{y} u(t)\right\rangle=0
$$


Consider now the $m-1$ fields $b^{1}, \ldots, b^{m-1}$ orthogonal to $b^{0}$ and satisfying (57). Let us denote by $\alpha^{0}, \alpha^{1}, \ldots, \alpha^{m-1}$ the coordinates of $a$ in the basis $b^{0}, b^{1}, \ldots, b^{m-1}$

$$
a(t, y)=\sum_{i=0}^{m-1} \alpha^{i}(t, y) b^{i}(y)
$$

Taking into account that $u(t) \in \operatorname{ker} T$ we have by (58) that $b^{i} \cdot \nabla_{y} u(t) \in \operatorname{ker} T, i \in$ $\{1, \ldots, m-1\}$ and $b^{0} \cdot \nabla_{y} u(t)=0$. Therefore we can write

$$
\left\langle a(t) \cdot \nabla_{y} u(t)\right\rangle=\sum_{i=0}^{m-1}\left\langle\alpha^{i}(t)\right\rangle b^{i} \cdot \nabla_{y} u(t)=\sum_{i=1}^{m-1}\left\langle\alpha^{i}(t)\right\rangle b^{i} \cdot \nabla_{y} u(t) .
$$

Actually the formula (66) holds true for any function in the kernel of $T$ saying that any transport operator $a \cdot \nabla_{y}$ reduces, by averaging along the flow of $b^{0}$ to another transport operator, denoted $\left\langle a \cdot \nabla_{y}\right\rangle$. If $a \cdot \nabla_{y}=\sum_{i=0}^{m-1} \alpha^{i} b^{i} \cdot \nabla_{y}$ is a linear combination of differential operators leaving invariant the kernel of $T$ then the effective operator by averaging is

$$
A \cdot \nabla_{y}=\left\langle a \cdot \nabla_{y}\right\rangle=\sum_{i=1}^{m-1}\left\langle\alpha^{i}\right\rangle b^{i} \cdot \nabla_{y}
$$

and therefore the model for the dominant term $u$ is given by

$$
\partial_{t} u+\sum_{i=1}^{m-1}\left\langle\alpha^{i}\right\rangle b^{i} \cdot \nabla_{y} u=0 .
$$

Remark 5.3 Assume that a, $A$ are smooth and verify

$$
\operatorname{div}_{y} a=0,\left\langle a \cdot \nabla_{y} u\right\rangle=A \cdot \nabla_{y} u, \quad u \in C_{c}^{1}\left(\mathbb{R}^{m}\right) \cap \operatorname{ker} T .
$$

Integrating with respect to $y \in \mathbb{R}^{m}$ we deduce that for any $u \in C_{c}^{1}\left(\mathbb{R}^{m}\right) \cap \operatorname{ker} T$

$$
\int_{\mathbb{R}^{m}} A \cdot \nabla_{y} u \mathrm{~d} y=\int_{\mathbb{R}^{m}}\left\langle a \cdot \nabla_{y} u\right\rangle \mathrm{d} y=\int_{\mathbb{R}^{m}} a \cdot \nabla_{y} u \mathrm{~d} y=-\int_{\mathbb{R}^{m}} u \operatorname{div}_{y} a \mathrm{~d} y=0 .
$$

Therefore we obtain

$$
\int_{\mathbb{R}^{m}} u\left\langle\operatorname{div}_{y} A\right\rangle \mathrm{d} y=\int_{\mathbb{R}^{m}} u \operatorname{div}_{y} A \mathrm{~d} y=-\int_{\mathbb{R}^{m}} A \cdot \nabla_{y} u \mathrm{~d} y=0
$$

implying that $\left\langle\operatorname{div}_{y} A\right\rangle=0$. In particular taking $\lambda^{0}=\lambda^{0}(y)$ such that $\left\langle\lambda^{0}\right\rangle=0, T \lambda^{0}=$ $\operatorname{div}_{y} A$ (which is possible because $\left\langle\operatorname{div}_{y} A\right\rangle=0$ ) we can replace the averaged transport operator $A \cdot \nabla_{y}$ by an equivalent one $A^{\prime} \cdot \nabla_{y}=A \cdot \nabla_{y}-\lambda^{0} b^{0} \cdot \nabla_{y}$

$$
\left\langle a \cdot \nabla_{y} u\right\rangle=A \cdot \nabla_{y} u=A^{\prime} \cdot \nabla_{y} u, \quad u \in C_{c}^{1}\left(\mathbb{R}^{m}\right) \cap \operatorname{ker} T
$$

such that

$$
\operatorname{div}_{y} A^{\prime}=\operatorname{div}_{y} A-\operatorname{div}_{y}\left(\lambda^{0} b^{0}\right)=\operatorname{div}_{y} A-T \lambda^{0}=0
$$


When considering the Vlasov equation (1) and the fields $b^{i}, i \in\{1,2,3,4,5\}$ in (60) we obtain

$$
\begin{gathered}
\alpha^{0}=-m \frac{\left(v_{\wedge} \cdot p\right)}{|p \wedge b(x)|^{2}}, \quad\left(\alpha^{1}, \alpha^{2}, \alpha^{3}\right)=\frac{p}{m}, \quad v_{\wedge}=\frac{E \wedge b}{B} \\
\alpha^{4}=-\frac{(p \cdot b(x))}{|p \wedge b(x)|}\left(\partial_{x} b: \frac{p \otimes p}{m}\right)+q E \cdot \frac{p-(p \cdot b(x)) b}{|p \wedge b(x)|}, \quad \alpha^{5}=\left(\partial_{x} b: \frac{p \otimes p}{m}\right)+q E \cdot b .
\end{gathered}
$$

The average of the above coefficients are

$$
\begin{gathered}
\left\langle\alpha^{0}\right\rangle=0,\left\langle\left(\alpha^{1}, \alpha^{2}, \alpha^{3}\right)\right\rangle=\frac{(p \cdot b(x))}{m} b \\
\left\langle\alpha^{4}\right\rangle=-\frac{(p \cdot b(x))|p \wedge b(x)|}{2 m} \operatorname{div}_{x} b,\left\langle\alpha^{5}\right\rangle=\frac{|p \wedge b(x)|^{2}}{2 m} \operatorname{div}_{x} b+q E \cdot b
\end{gathered}
$$

and therefore the average of the transport operator $\frac{p}{m} \cdot \nabla_{x}+q E \cdot \nabla_{p}$ is

$$
\left\langle\frac{p}{m} \cdot \nabla_{x}+q E \cdot \nabla_{p}\right\rangle=\frac{(p \cdot b(x))}{m} b \cdot \nabla_{x}+\left(q(E \cdot b) b+\omega(x, p){ }^{\perp} p\right) \cdot \nabla_{p}
$$

with

$$
\omega(x, p)=\frac{|p \wedge b(x)|}{2 m} \operatorname{div}_{x} b-\frac{(p \cdot b(x))}{m}\left(\partial_{x} b b \cdot \frac{p}{|p \wedge b(x)|}\right) .
$$

We have retrieved the limit model (31).

Remark 5.4 It is also possible to determine the average transport operator $A \cdot \nabla_{y}=$ $\left\langle a \cdot \nabla_{y}\right\rangle$ by imposing (66) for any prime integral $\psi_{i}, i \in\{1,2, \ldots, m-1\}$ together with the condition $A \cdot b^{0}=0$. It is easily seen that any prime integral for a which belongs to the kernel of $T$ is also a prime integral for $A$. Indeed, if $a \cdot \nabla_{y} I=0$ then $A$. $\nabla_{y} I=\left\langle a \cdot \nabla_{y} I\right\rangle=0$. In particular $|p|^{2} / 2 m+q \phi(t, x) \in \operatorname{ker} \mathcal{T}$ is a prime integral for $\left((p \cdot b) b / m, q(E \cdot b) b+\omega(x, p)^{\perp} p\right)$ if $E(t, x)=-\nabla_{x} \phi(t, x)$.

In the sequel we intend to take into account the first order corrections $u^{1}$, that is, write a model whose solution coincides with $u+\varepsilon u^{1}$ up to $\mathcal{O}\left(\varepsilon^{2}\right)$. By using the orthogonal decomposition in $L^{2}\left(\mathbb{R}^{m}\right)$

$$
u^{1}=v^{1}+w^{1}, \quad T v^{1}=0, \quad\left\langle w^{1}\right\rangle=0
$$

we obtain from $(63),(65)$

$$
\begin{aligned}
-T w^{1} & =\partial_{t} u+a \cdot \nabla_{y} u \\
& =\partial_{t} u+a \cdot \nabla_{y} u-\left\langle\partial_{t} u+a \cdot \nabla_{y} u\right\rangle \\
& =\sum_{i=1}^{m-1}\left(\alpha^{i}-\left\langle\alpha^{i}\right\rangle\right) b^{i} \cdot \nabla_{y} u .
\end{aligned}
$$


Therefore the zero average contribution of $u^{1}$ is given by

$$
w^{1}=-\tilde{A} \cdot \nabla_{y} u, \quad \tilde{A}=\sum_{i=1}^{m-1} \beta^{i} b^{i}
$$

where for any $i \in\{1,2, \ldots, m-1\}, \beta^{i}$ solves

$$
T \beta^{i}=\alpha^{i}-\left\langle\alpha^{i}\right\rangle \in \operatorname{ker}\langle\cdot\rangle,\left\langle\beta^{i}\right\rangle=0 .
$$

Applying the average operator in (64) yields

$$
\partial_{t} v^{1}+A \cdot \nabla_{y} v^{1}+\left\langle a \cdot \nabla_{y} w^{1}\right\rangle=0
$$

and combining (65), (73) leads to

$$
\partial_{t}\left(u+\varepsilon u^{1}\right)+A \cdot \nabla_{y}\left(u+\varepsilon u^{1}\right)+\varepsilon\left(\left\langle a \cdot \nabla_{y} w^{1}\right\rangle-\partial_{t} w^{1}-A \cdot \nabla_{y} w^{1}\right)=0 .
$$

Replacing the time derivative in terms of space derivatives thanks to $\partial_{t} u=-A \cdot \nabla_{y} u$ we transform the time derivative of $w^{1}$ as follows

$$
-\partial_{t} w^{1}=\partial_{t} \tilde{A} \cdot \nabla_{y} u-\tilde{A} \cdot \nabla_{y}\left(A \cdot \nabla_{y} u\right)
$$

The equation (74) can be written in the form

$$
\partial_{t}\left(u+\varepsilon u^{1}\right)+A \cdot \nabla_{y}\left(u+\varepsilon u^{1}\right)+\varepsilon\left(\partial_{t} \tilde{A} \cdot \nabla_{y} u+R(u)\right)=0
$$

where

$$
R(u)=\left\langle a \cdot \nabla_{y} w^{1}\right\rangle-A \cdot \nabla_{y} w^{1}-\tilde{A} \cdot \nabla_{y}\left(A \cdot \nabla_{y} u\right) .
$$

We need to express $R(u)$ in terms of $u$ in view of (72). Eventually $R(u)$ contains second order derivatives of $u$. We will see that all second order derivatives cancel. Moreover we will show that $R(u)$ reduces to a first order differential operator. Let us split the computations into several steps.

Proposition 5.6 Assume that $\operatorname{div}_{y} a=0$. For any function $u \in \operatorname{ker} T$ we have

$$
\left\langle a \cdot \nabla_{y} w^{1}\right\rangle=\sum_{j=1}^{m-1}\left[\sum_{i=1}^{m-1}\left\{b^{i} \cdot \nabla_{y}\left\langle\beta^{i} T \beta^{j}\right\rangle+\left\langle\operatorname{div}_{y} b^{i}\right\rangle\left\langle\beta^{i} T \beta^{j}\right\rangle\right\}\right] b^{j} \cdot \nabla_{y} u .
$$


Proof. By Proposition 5.5 we can write

$$
\begin{aligned}
\left\langle a \cdot \nabla_{y} w^{1}\right\rangle & =\left\langle\sum_{i=0}^{m-1} \alpha^{i} b^{i} \cdot \nabla_{y} w^{1}\right\rangle \\
& =\left\langle\sum_{i=0}^{m-1} b^{i} \cdot \nabla_{y}\left(\alpha^{i} w^{1}\right)\right\rangle-\left\langle\sum_{i=0}^{m-1}\left(b^{i} \cdot \nabla_{y} \alpha^{i}\right) w^{1}\right\rangle \\
& =\sum_{i=0}^{m-1} b^{i} \cdot \nabla_{y}\left\langle\alpha^{i} w^{1}\right\rangle-\left\langle\sum_{i=0}^{m-1}\left(\alpha^{i} w^{1}-\left\langle\alpha^{i} w^{1}\right\rangle\right) \operatorname{div}_{y} b^{i}\right\rangle-\sum_{i=0}^{m-1}\left\langle\left(b^{i} \cdot \nabla_{y} \alpha^{i}\right) w^{1}\right\rangle \\
& =\sum_{i=0}^{m-1} b^{i} \cdot \nabla_{y}\left\langle\alpha^{i} w^{1}\right\rangle+\sum_{i=0}^{m-1}\left\langle\alpha^{i} w^{1}\right\rangle\left\langle\operatorname{div}_{y} b^{i}\right\rangle-\left\langle w^{1} \operatorname{div}_{y} a\right\rangle \\
& =\sum_{i=1}^{m-1} b^{i} \cdot \nabla_{y}\left\langle\alpha^{i} w^{1}\right\rangle+\sum_{i=1}^{m-1}\left\langle\alpha^{i} w^{1}\right\rangle\left\langle\operatorname{div}_{y} b^{i}\right\rangle .
\end{aligned}
$$

In the last equality we have taken into account that $\left\langle\alpha^{0} w^{1}\right\rangle \in \operatorname{ker} T$ and $\operatorname{div}_{y} b^{0}$. Since $\left\langle w^{1}\right\rangle=0$ we have for any $i \in\{1, \ldots, m-1\}$

$$
\begin{aligned}
\left\langle\alpha^{i} w^{1}\right\rangle & =\left\langle w^{1} T \beta^{i}\right\rangle \\
& =-\left\langle\beta^{i} T w^{1}\right\rangle \\
& =\sum_{j=1}^{m-1}\left\langle\beta^{i} T \beta^{j}\right\rangle b^{j} \cdot \nabla_{y} u .
\end{aligned}
$$

Notice that the matrix $\left(\left\langle\beta^{i} T \beta^{j}\right\rangle\right)_{i, j}$ is skew-symmetric

$$
\left\langle\beta^{i} T \beta^{j}\right\rangle+\left\langle\beta^{j} T \beta^{i}\right\rangle=\left\langle T\left(\beta^{i} \beta^{j}\right)\right\rangle=0, \quad i, j \in\{1, \ldots, m-1\} .
$$

Another key point is that for any $i, j \in\{1, \ldots, m-1\}$ the operators $b^{i} \cdot \nabla_{y}, b^{j} \cdot \nabla_{y}$ are commuting on functions of $\operatorname{ker} T$. Indeed if $u=v\left(\psi_{1}, \ldots, \psi_{m-1}\right) \in \operatorname{ker} T$ we have cf. (58)

$$
b^{i} \cdot \nabla_{y} u=\partial_{\psi_{i}} v(\psi(y)), \quad b^{j} \cdot \nabla_{y} u=\partial_{\psi_{j}} v(\psi(y)) .
$$

It follows that

$$
b^{i} \cdot \nabla_{y}\left(b^{j} \cdot \nabla_{y} u\right)-b^{j} \cdot \nabla_{y}\left(b^{i} \cdot \nabla_{y} u\right)=\partial_{\psi_{i} \psi_{j}}^{2} v(\psi(y))-\partial_{\psi_{j} \psi_{i}}^{2} v(\psi(y))=0 .
$$

Combining the skew-symmetry of $\left(\left\langle\beta^{i} T \beta^{j}\right\rangle\right)_{i, j}$ and the symmetry of $\left(b^{i} \cdot \nabla_{y}\left(b^{j} \cdot \nabla_{y} u\right)\right)_{i, j}$ we deduce that

$$
\sum_{i=1}^{m-1} b^{i} \cdot \nabla_{y}\left\langle\alpha^{i} w^{1}\right\rangle=\sum_{i=1}^{m-1} b^{i} \cdot \nabla_{y}\left[\sum_{j=1}^{m-1}\left\langle\beta^{i} T \beta^{j}\right\rangle b^{j} \cdot \nabla_{y} u\right]=\sum_{j=1}^{m-1}\left[\sum_{i=1}^{m-1} b^{i} \cdot \nabla_{y}\left\langle\beta^{i} T \beta^{j}\right\rangle\right] b^{j} \cdot \nabla_{y} u .
$$


Therefore the term $\left\langle a \cdot \nabla_{y} w^{1}\right\rangle$ can be written

$$
\left\langle a \cdot \nabla_{y} w^{1}\right\rangle=\sum_{j=1}^{m-1}\left[\sum_{i=1}^{m-1}\left\{b^{i} \cdot \nabla_{y}\left\langle\beta^{i} T \beta^{j}\right\rangle+\left\langle\operatorname{div}_{y} b^{i}\right\rangle\left\langle\beta^{i} T \beta^{j}\right\rangle\right\}\right] b^{j} \cdot \nabla_{y} u .
$$

Proposition 5.7 For any function $u \in \operatorname{ker} T$ we have

$$
-A \cdot \nabla_{y} w^{1}-\tilde{A} \cdot \nabla_{y}\left(A \cdot \nabla_{y} u\right)=[A, \tilde{A}] \cdot \nabla_{y} u
$$

where $[A, \tilde{A}]$ stands for the Poisson bracket between the fields $A=\sum_{i=1}^{m-1}\left\langle\alpha^{i}\right\rangle b^{i}$ and $\tilde{A}=\sum_{i=1}^{m-1} \beta^{i} b^{i}$.

Proof. Obviously we have

$$
-A \cdot \nabla_{y} w^{1}-\tilde{A} \cdot \nabla_{y}\left(A \cdot \nabla_{y} u\right)=A \cdot \nabla_{y}\left(\tilde{A} \cdot \nabla_{y} u\right)-\tilde{A} \cdot \nabla_{y}\left(A \cdot \nabla_{y} u\right)=[A, \tilde{A}] \cdot \nabla_{y} u
$$

Proposition 5.8 Assume that $\operatorname{div}_{y} a=0$. Then for any function $u \in \operatorname{ker} T$ we have

$$
R(u)=\sum_{j=1}^{m-1}\left[\sum_{i=1}^{m-1}\left\{b^{i} \cdot \nabla_{y}\left\langle\beta^{i} T \beta^{j}\right\rangle+\left\langle\operatorname{div}_{y} b^{i}\right\rangle\left\langle\beta^{i} T \beta^{j}\right\rangle\right\}\right] b^{j} \cdot \nabla_{y} u+[A, \tilde{A}] \cdot \nabla_{y} u .
$$

Remark 5.5 If $I(t) \in \operatorname{ker} T$ is a prime integral for $a(t)=\sum_{i=0}^{m-1} \alpha^{i}(t) b^{i}$ therefore $I(t)$ is also a prime integral for $A(t)=\sum_{i=1}^{m-1}\left\langle\alpha^{i}(t)\right\rangle b^{i}$ cf. Remark 5.4 and thus the corresponding function $w^{1}$ vanishes, because $-T w^{1}=a(t) \cdot \nabla_{y} I(t)-A(t) \cdot \nabla_{y} I(t)=0$. We deduce by Proposition 5.8 that $I(t)$ is a prime integral for $R$ i.e., $R(I(t))=0$.

Coming back in $(75)$ we deduce that $u+\varepsilon u^{1}$ solves $\partial_{t}\left(u+\varepsilon u^{1}\right)+A \cdot \nabla_{y}\left(u+\varepsilon u^{1}\right)+\varepsilon\left[\partial_{t} \tilde{A} \cdot \nabla_{y}\left(u+\varepsilon u^{1}\right)+R\left(u+\varepsilon u^{1}\right)\right]=\varepsilon^{2}\left[\partial_{t} \tilde{A} \cdot \nabla_{y} u^{1}+R\left(u^{1}\right)\right]$. Therefore we expect that the function $\tilde{u}^{\varepsilon}$ solving

$$
\begin{gathered}
\partial_{t} \tilde{u}^{\varepsilon}+\left(A+\varepsilon A^{1}\right) \cdot \nabla_{y} \tilde{u}^{\varepsilon}=0 \\
A^{1}=\partial_{t} \tilde{A}+\sum_{j=1}^{m-1}\left[\sum_{i=1}^{m-1}\left\{b^{i} \cdot \nabla_{y}\left\langle\beta^{i} T \beta^{j}\right\rangle+\left\langle\operatorname{div}_{y} b^{i}\right\rangle\left\langle\beta^{i} T \beta^{j}\right\rangle\right\}\right] b^{j}+[A, \tilde{A}]
\end{gathered}
$$


will be a second order approximation for $u+\varepsilon u^{1}$

$$
\tilde{u}^{\varepsilon}=u+\varepsilon u^{1}+\mathcal{O}\left(\varepsilon^{2}\right) .
$$

Consequently, motivated by the ansatz $u^{\varepsilon}=u+\varepsilon u^{1}+\varepsilon^{2} u^{2}+\ldots$, the solution of $(77)$ will approximate the solution of (61) up to a second order term

$$
\tilde{u}^{\varepsilon}=u^{\varepsilon}+\mathcal{O}\left(\varepsilon^{2}\right)
$$

\subsection{Derivation of the gyrokinetic Vlasov equation}

This section is devoted to the explicit computation of the second order model (77) which corresponds to the Vlasov equation (1) with strong magnetic field. We already know that the transport operator $A \cdot \nabla_{x, p}$ has the form

$$
A \cdot \nabla_{x, p}=\frac{(p \cdot b)}{m} b \cdot \nabla_{x}+\left(q(E \cdot b) b+\omega(x, p)^{\perp} p\right) \cdot \nabla_{p}
$$

It remains to identify the transport operator $A^{1} \cdot \nabla_{x, p}$. We need the following lemma, whose proof is left to the reader.

Lemma 5.3 We have the formula

$$
\begin{gathered}
\mathcal{T}^{-1}(p-\langle p\rangle)=-\frac{p \wedge b}{\omega_{c}(x)} \\
\mathcal{T}^{-1}(p \otimes p-\langle p \otimes p\rangle)=-\frac{p \wedge b}{\omega_{c}} \otimes\left[\frac{3}{4}(p \cdot b) b+\frac{p}{4}\right]-\left[\frac{3}{4}(p \cdot b) b+\frac{p}{4}\right] \otimes \frac{p \wedge b}{\omega_{c}} .
\end{gathered}
$$

Proposition 5.9 The transport operator $\tilde{A} \cdot \nabla_{x, p}=\tilde{A}_{x} \cdot \nabla_{x}+\tilde{A}_{p} \cdot \nabla_{p}$ is given by

$$
\tilde{A}_{x}=-\frac{p \wedge b}{m \omega_{c}}
$$

$\tilde{A}_{p}=\frac{m\left(v_{\wedge} \cdot p\right)}{|p \wedge b(x)|} \frac{p-(p \cdot b(x)) b}{|p \wedge b(x)|}+\left[{ }^{t} \partial_{x} b p \cdot(p \wedge b)+\omega_{c}\left(\partial_{x} b: \mathcal{T}^{-1}(p \otimes p-\langle p \otimes p\rangle)\right] \frac{{ }^{\perp} p}{m \omega_{c}|p \wedge b|}\right.$.

In particular we have

$$
\partial_{t} \tilde{A} \cdot \nabla_{x, p}=\frac{m\left(\partial_{t} v_{\wedge} \cdot p\right)}{|p \wedge b(x)|} \frac{p-(p \cdot b(x)) b}{|p \wedge b(x)|} \cdot \nabla_{p}, \quad v_{\wedge}=\frac{E \wedge b}{B}
$$


Proof. Using (78) and the expressions of the coefficients $\alpha^{i},\left\langle\alpha^{i}\right\rangle, i \in\{1, \ldots 5\}$ computed before cf. (68), (69), (70), (71) we obtain

$$
\begin{gathered}
\left(\beta^{1}, \beta^{2}, \beta^{3}\right)=\mathcal{T}^{-1} \frac{p-\langle p\rangle}{m}=-\frac{p \wedge b}{m \omega_{c}} \\
\beta^{4}=-\frac{(p \cdot b(x))}{|p \wedge b(x)|}\left(\partial_{x} b: \mathcal{T}^{-1} \frac{p \otimes p-\langle p \otimes p\rangle}{m}\right)+m \frac{v_{\wedge} \cdot p}{|p \wedge b(x)|} \\
\beta^{5}=\left(\partial_{x} b: \mathcal{T}^{-1} \frac{p \otimes p-\langle p \otimes p\rangle}{m}\right) .
\end{gathered}
$$

Therefore thanks to (60) we deduce that

$$
\tilde{A}_{x}=-\frac{p \wedge b}{m \omega_{c}(x)}
$$

$\tilde{A}_{p}=\left[{ }^{t} \partial_{x} b p \cdot(p \wedge b)+\omega_{c}\left(\partial_{x} b: \mathcal{T}^{-1}(p \otimes p-\langle p \otimes p\rangle)\right] \frac{{ }^{\perp} p}{m \omega_{c}|p \wedge b|}+m \frac{v_{\wedge} \cdot p}{|p \wedge b|} \frac{p-(p \cdot b) b}{|p \wedge b|}\right.$ and (80) follows.

Based on the above considerations we complete now the regularity result used in the proof of Theorem 4.2 .

Proposition 5.10 We suppose that $E \in L_{\mathrm{loc}}^{\infty}\left(\mathbb{R}_{+} ; W^{1, \infty}\left(\mathbb{R}^{3}\right)\right)^{3}, \partial_{t} E \in L_{\mathrm{loc}}^{\infty}\left(\mathbb{R}_{+} ; L^{\infty}\left(\mathbb{R}^{3}\right)\right)^{3}$, $b \in W^{2, \infty}\left(\mathbb{R}^{3}\right)^{3}, B \in W^{1, \infty}\left(\mathbb{R}^{3}\right), \operatorname{div}_{x}(B b)=0$ and $\inf _{x \in \mathbb{R}^{3}} B(x)>0$. Let $f^{\text {in }} \in$ ker $\mathcal{T} \cap C_{c}^{2}\left(\mathbb{R}^{3} \times \mathbb{R}^{3}\right)$ verifying

$$
f^{\text {in }}(x, p)=0, \quad(x, p) \in \mathbb{R}^{3} \times \mathbb{R}^{3}, \quad|p \wedge b(x)| \leq r^{\text {in }}
$$

for some $r^{\text {in }}>0$. We denote by $f=f(t, x, p)$ the solution of the limit model $\partial_{t} f+b(x) \otimes b(x) \frac{p}{m} \cdot \nabla_{x} f+\left(q b(x) \otimes b(x) E+\omega(x, p){ }^{\perp} p\right) \cdot \nabla_{p} f=0, \quad(p \wedge b) \cdot \nabla_{p} f=0$ with the initial condition $f(0, x, p)=f^{\text {in }}(x, p),(x, p) \in \mathbb{R}^{3} \times \mathbb{R}^{3}$. For any $t \in \mathbb{R}_{+}$we consider $h(t) \in \mathrm{D}(\mathcal{T})$ the unique function satisfying

$$
\partial_{t} f+\frac{p}{m} \cdot \nabla_{x} f+q E(t, x) \cdot \nabla_{p} f+\mathcal{T} h(t)=0,\langle h(t)\rangle=0 .
$$

Therefore for any $T>0$ the function $h$ belongs to $W^{1, \infty}\left([0, T] \times \mathbb{R}^{3} \times \mathbb{R}^{3}\right)$ and has compact support. In particular $\partial_{t} h+\frac{p}{m} \cdot \nabla_{x} h+q E(t, x) \cdot \nabla_{p} h \in L^{\infty}\left([0, T] ; L^{2}\left(\mathbb{R}^{3} \times \mathbb{R}^{3}\right)\right)$. 
Proof. As in the proof of Theorem 4.2, the regularity of the electro-magnetic field guarantees that $f \in W^{2, \infty}\left([0, T] \times \mathbb{R}^{3} \times \mathbb{R}^{3}\right)$. Moreover, the compactness of the support of $f^{\text {in }}$ implies the compactness of the support of $f(t)$, uniformly for $t \in[0, T]$. We have

$$
-\mathcal{T} h(t)=\sum_{i=1}^{5}\left(\alpha^{i}-\left\langle\alpha^{i}\right\rangle\right) b^{i} \cdot \nabla_{x, p} f(t)=\sum_{i=1}^{5} \mathcal{T} \beta^{i} b^{i} \cdot \nabla_{x, p} f(t)
$$

saying that

$$
-h(t)=\sum_{i=1}^{5} \beta^{i} b^{i} \cdot \nabla_{x, p} f(t)=\tilde{A} \cdot \nabla_{x, p} f(t) .
$$

Obviously $h$ has compact support and by Lemma 5.3, Proposition 5.9 we deduce that the components of the field $\tilde{A}$ are locally Lipschitz in $\left\{(t, x, p) \in[0, T] \times \mathbb{R}^{3} \times \mathbb{R}^{3}\right.$ : $\left.|p \wedge b(x)| \geq r^{\text {in }} \sqrt{\inf B / \sup B}\right\}$. Our conclusion follows immediately by observing that the invariance of the magnetic moment $\mu=|p \wedge b|^{2} / 2 m B$ ensures

$$
\operatorname{supp} f(t) \subset\left\{(t, x, p) \in[0, T] \times \mathbb{R}^{3} \times \mathbb{R}^{3}:|p \wedge b(x)| \geq r \sqrt{\frac{\operatorname{in}}{\sup B}}\right\}, t \in \mathbb{R}_{+} .
$$

We need to compute the matrix $\left(\left\langle\beta^{i} \mathcal{T} \beta^{j}\right\rangle\right)_{1 \leq i, j \leq 5}$. The following formula will be used.

Lemma 5.4 For any $k \in\{1,2,3\}$ we have

$$
\left\langle p_{k} p \otimes p\right\rangle=(p \cdot b) b_{k}\left[(p \cdot b)^{2}-\frac{3}{2}|p \wedge b|^{2}\right] b \otimes b+\frac{(p \cdot b)|p \wedge b|^{2}}{2}\left[b_{k} I+b \otimes e_{k}+e_{k} \otimes b\right] .
$$

Proposition 5.11 The elements of the matrix $\left(\left\langle\beta^{i} \mathcal{T} \beta^{j}\right\rangle\right)_{1 \leq i, j \leq 5}$ are given by

$$
\left(\begin{array}{ccc}
\frac{|p \wedge b|^{2}}{2 m^{2} \omega_{c}} M[b] & -\frac{|p \wedge b|}{2}\left(v_{\wedge}+v_{\mathrm{CD}}\right) & \frac{(p \cdot b)|p \wedge b|^{2}}{2 m^{2} \omega_{c}} b \wedge \partial_{x} b b \\
\frac{|p \wedge b|}{2} t\left(v_{\wedge}+v_{\mathrm{CD}}\right) & 0 & \frac{(p \cdot b)|p \wedge b|}{2 B} E \cdot\left(b \wedge \partial_{x} b b\right) \\
-{ }^{t}\left[\frac{(p \cdot b)|p \wedge b|^{2}}{2 m^{2} \omega_{c}} b \wedge \partial_{x} b b\right] & -\frac{(p \cdot b)|p \wedge b|}{2 B} E \cdot\left(b \wedge \partial_{x} b b\right) & 0
\end{array}\right)
$$

where $M[b]$ is the matrix of the linear map $p \rightarrow b \wedge p$

$$
M[b]=\left(\begin{array}{ccc}
0 & -b_{3} & b_{2} \\
b_{3} & 0 & -b_{1} \\
-b_{2} & b_{1} & 0
\end{array}\right)
$$

and $v_{\wedge}=\frac{E \wedge b}{B}, v_{\mathrm{CD}}=\frac{(p \cdot b)^{2}}{m^{2} \omega_{c}} b \wedge \partial_{x} b b$ 
Proof. Recall that the matrix $\left(\left\langle\beta^{i} \mathcal{T} \beta^{j}\right\rangle\right)_{1 \leq i, j \leq 5}$ is skew-symmetric and

$$
\left\langle\beta^{i} \mathcal{T} \beta^{j}\right\rangle=\left\langle\beta^{i} \alpha^{j}\right\rangle, \quad i, j \in\{1, \ldots, 5\}
$$

After computations we deduce that the matrix $\langle\beta \otimes \alpha\rangle$ is given by (82).

Thanks to Proposition 5.6 we obtain the following explicit expression for the term $\left\langle a \cdot \nabla_{y} w^{1}\right\rangle$.

Proposition 5.12 For any function $u \in \operatorname{ker} \mathcal{T}$ we have

$$
\begin{aligned}
\left\langle a \cdot \nabla_{y} w^{1}\right\rangle & =\left(v_{\wedge}+v_{\mathrm{GD}}+v_{\mathrm{CD}}+v_{\mathrm{RD}}\right) \cdot \nabla_{x} u-\frac{{ }^{t} \partial_{x} b p}{|p \wedge b|} \cdot\left(v_{\wedge}+v_{\mathrm{GD}}+v_{\mathrm{CD}}+v_{\mathrm{RD}}\right)^{\perp} p \cdot \nabla_{p} u \\
& +\frac{(p \cdot b)|p \wedge b|}{2 m^{2} \omega_{c}} B \operatorname{div}_{x}\left(\frac{b \wedge \partial_{x} b b}{B}\right){ }^{\perp} p \cdot \nabla_{p} u \\
& +\left(\partial_{x} b b \cdot v_{\wedge}\right)\left(\frac{3}{2}(p \cdot b) b-\frac{p}{2}\right) \cdot \nabla_{p} u-\left(\operatorname{div}_{x} v_{\wedge}\right) \frac{p-(p \cdot b) b}{2} \cdot \nabla_{p} u
\end{aligned}
$$

where $v_{\wedge}, v_{\mathrm{GD}}, v_{\mathrm{CD}}, v_{\mathrm{RD}}$ are the electric cross field drift, the magnetic gradient drift, the magnetic curvature drift and the magnetic rotational drift respectively

$v_{\wedge}=\frac{E \wedge b}{B}, \quad v_{\mathrm{GD}}=\frac{|p \wedge b|^{2}}{2 m^{2} \omega_{c}} \frac{b \wedge \nabla_{x} B}{B}, \quad v_{\mathrm{CD}}=\frac{(p \cdot b)^{2}}{m^{2} \omega_{c}} b \wedge \partial_{x} b b, \quad v_{\mathrm{RD}}=\frac{|p \wedge b|^{2}}{2 m^{2} \omega_{c}}\left(b \cdot \operatorname{rot}_{x} b\right) b$.

Proof. The entries of the matrix $\left(\left\langle\beta^{i} \mathcal{T} \beta^{j}\right\rangle\right)_{1 \leq i, j \leq 5}$ belong to the kernel of $\mathcal{T}$. Thus the terms $b^{i} \cdot \nabla_{x, p}\left\langle\beta^{i} \mathcal{T} \beta^{j}\right\rangle$ can be easily computed since $b^{i} \cdot \nabla_{x, p}=\partial_{\psi_{i}}$ for any $i \in\{1, \ldots, 5\}$ cf. (58). Notice also that $\left\langle\operatorname{div}_{x, p} b^{i}\right\rangle=0$ for $i \in\{1,2,3,5\}$ and $\left\langle\operatorname{div}_{x, p} b^{4}\right\rangle=1 /|p \wedge b|$ implying that

$$
\left\langle a \cdot \nabla_{y} w^{1}\right\rangle=\sum_{j=1}^{5}\left[\sum_{i=1}^{5} b^{i} \cdot \nabla_{x, p}\left\langle\beta^{i} \mathcal{T} \beta^{j}\right\rangle+\frac{\left\langle\beta^{4} \mathcal{T} \beta^{j}\right\rangle}{|p \wedge b|}\right] b^{j} \cdot \nabla_{x, p} u=\sum_{j=1}^{5} C^{j} b^{j} \cdot \nabla_{x, p} u .
$$

After computations one gets

$$
\begin{gathered}
\left(C^{1}, C^{2}, C^{3}\right)=v_{\wedge}+v_{\mathrm{GD}}+v_{\mathrm{CD}}+v_{\mathrm{RD}} \\
C^{4}=-\frac{(p \cdot b)^{2}|p \wedge b|}{2 m^{2} \omega_{c}} B(x) \operatorname{div}_{x}\left(\frac{b \wedge \partial_{x} b b}{B}\right)-\frac{|p \wedge b|}{2}\left[\left(v_{\wedge} \cdot \partial_{x} b b\right)+\operatorname{div}_{x} v_{\wedge}\right] \\
C^{5}=\frac{(p \cdot b)|p \wedge b|^{2}}{2 m^{2} \omega_{c}} B(x) \operatorname{div}_{x}\left(\frac{b \wedge \partial_{x} b b}{B}\right)+(p \cdot b)\left(v_{\wedge} \cdot \partial_{x} b b\right)
\end{gathered}
$$

and the conclusion follows. 
Finally combining Propositions 5.9, 5.12 we obtain

Theorem 5.2 The second order model (77) which corresponds to the Vlasov equation (1) with strong magnetic fields is

$$
\partial_{t} \tilde{f}^{\varepsilon}+\left(A_{x}+\varepsilon A_{x}^{1}\right) \cdot \nabla_{x} \tilde{f}^{\varepsilon}+\left(A_{p}+\varepsilon A_{p}^{1}\right) \cdot \nabla_{p} \tilde{f}^{\varepsilon}=0
$$

where

$$
\begin{gathered}
A_{x}=\frac{(p \cdot b)}{m} b, \quad A_{p}=q(E \cdot b) b+\omega(x, p){ }^{\perp} p, \varepsilon \tilde{A}_{x}=-\frac{p \wedge b}{m \omega_{c}^{\varepsilon}} \\
\varepsilon \tilde{A}_{p}=m \frac{v_{\wedge}^{\varepsilon} \cdot p}{|p \wedge b|} \frac{p-(p \cdot b) b}{|p \wedge b|}+\left[{ }^{t} \partial_{x} b p \cdot(p \wedge b)+\omega_{c}\left(\partial_{x} b: \mathcal{T}^{-1}(p \otimes p-\langle p \otimes p\rangle)\right] \frac{{ }^{\perp} p}{m \omega_{c}^{\varepsilon}|p \wedge b|}\right. \\
\varepsilon A_{x}^{1}=v_{\wedge}^{\varepsilon}+v_{\mathrm{GD}}^{\varepsilon}+v_{\mathrm{CD}}^{\varepsilon}+v_{\mathrm{RD}}^{\varepsilon}+[A, \varepsilon \tilde{A}]_{x} \\
\varepsilon A_{p}^{1}=m \frac{\partial_{t} v_{\wedge}^{\varepsilon} \cdot p}{|p \wedge b|} \frac{p-(p \cdot b) b}{|p \wedge b|}-\frac{{ }^{t} \partial_{x} b p}{|p \wedge b|} \cdot\left(v_{\wedge}^{\varepsilon}+v_{\mathrm{GD}}^{\varepsilon}+v_{\mathrm{CD}}^{\varepsilon}+v_{\mathrm{RD}}^{\varepsilon}\right){ }^{\perp} p \\
+\frac{(p \cdot b)|p \wedge b|}{2 m^{2} \omega_{c}^{\varepsilon}} B^{\varepsilon} \operatorname{div}_{x}\left(\frac{b \wedge \partial_{x} b b}{B^{\varepsilon}}\right){ }^{\perp} p \\
+\left(\partial_{x} b b \cdot v_{\wedge}^{\varepsilon}\right)\left(\frac{3}{2}(p \cdot b) b-\frac{p}{2}\right)-\left(\operatorname{div}_{x} v_{\wedge}^{\varepsilon}\right) \frac{p-(p \cdot b) b}{2}+[A, \varepsilon \tilde{A}]_{p}
\end{gathered}
$$

and

$$
\begin{gathered}
B^{\varepsilon}=\frac{B}{\varepsilon}, \omega_{c}^{\varepsilon}=\frac{q B^{\varepsilon}}{m} \\
v_{\wedge}^{\varepsilon}=\frac{E \wedge b}{B^{\varepsilon}}, v_{\mathrm{GD}}^{\varepsilon}=\frac{|p \wedge b|^{2}}{2 m^{2} \omega_{c}^{\varepsilon}} \frac{b \wedge \nabla_{x} B^{\varepsilon}}{B^{\varepsilon}}, \quad v_{\mathrm{CD}}^{\varepsilon}=\frac{(p \cdot b)^{2}}{m^{2} \omega_{c}^{\varepsilon}} b \wedge \partial_{x} b b, \quad v_{\mathrm{RD}}^{\varepsilon}=\frac{|p \wedge b|^{2}}{2 m^{2} \omega_{c}^{\varepsilon}}\left(b \cdot \operatorname{rot}_{x} b\right) b .
\end{gathered}
$$

\section{References}

[1] N.N. Bogoliubov, Y.A. Mitropolsky, Asymptotic methods in the theory of nonlinear oscillations, Gordon and Breach Sciences Publishers, New York, 1961.

[2] M. Bostan, The Vlasov-Poisson system with strong external magnetic field. Finite Larmor radius regime, Asymptot. Anal., 61(2009) 91-123.

[3] M. Bostan, The Vlasov-Maxwell system with strong initial magnetic field. Guiding-center approximation, SIAM J. Multiscale Model. Simul. 6(2007) 10261058.

[4] M. Bostan, Transport equations with disparate advection fields. Application to the gyrokinetic models in plasma physics, research report INRIA. 
[5] M. Bostan, T. Goudon, High-electric-field limit for the Vlasov-Maxwell-FokkerPlanck system, Ann. Inst. H. Poincaré, Anal. Non Linéaire, 25(2008) 1221-1251.

[6] Y. Brenier, Convergence of the Vlasov-Poisson system to the incompressible Euler equations, Comm. Partial Differential Equations 25(2000) 737-754.

[7] Y. Brenier, N. Mauser, M. Puel, Incompressible Euler and e-MHD as scaling limits of the Vlasov-Maxwell system, Commun. Math. Sci. 1(2003) 437-447.

[8] H. Brezis, Analyse fonctionnelle. Théorie et applications, Masson 1983.

[9] A.J. Brizard, T.S. Hahm, Foundations of nonlinear gyrokinetic theory, Rev. Modern Phys., 79(2007) 421-468.

[10] E. Frénod, E. Sonnendrücker, Homogenization of the Vlasov equation and of the Vlasov-Poisson system with strong external magnetic field, Asymptotic Anal. 18(1998) 193-213.

[11] E. Frénod, E. Sonnendrücker, The finite Larmor radius approximation, SIAM J. Math. Anal. 32(2001) 1227-1247.

[12] F. Golse, L. Saint-Raymond, The Vlasov-Poisson system with strong magnetic field, J. Math. Pures Appl. 78(1999) 791-817.

[13] F. Golse, L. Saint-Raymond, The Vlasov-Poisson system with strong magnetic field in quasineutral regime, Math. Models Methods Appl. Sci. 13(2003) 661-714.

[14] V. Grandgirard, M. Brunetti, P. Bertrand, N. Besse, X. Garbet, P. Ghendrih, G. Manfredi, Y. Sarazin, O. Sauter, E. Sonnendrücker, J. Vaclavik, L. Villard, A drift-kinetic semi-Lagrangian 4D code for ion turbulence simulation, J. Comput. Phys. 217(2006) 395-423.

[15] R.D. Hazeltine, J.D. Meiss, Plasma confinement, Dover Publications, Inc. Mineola, New York, 2003.

[16] R. G. Littlejohn, A guiding center Hamiltonian : A new approach, J. Math. Phys. 20(1979) 2445-2458. 
[17] R. G. Littlejohn, Hamiltonian formulation of guiding center motion, Phys. Fluids 24(1981) 1730-1749.

[18] P. Morel, E. Gravier, N. Besse, A. Ghizzo, P. Bertrand, The water bag model and gyrokinetic applications, Commun. Nonlinear Sci. Numer. Simul. 13(2008) $11-17$.

[19] M. Reed, B. Simon, Methods of Modern Mathematical Physics, Vol. I, Functional Analysis, Academic Press 1980. 